\title{
Classical Path Methods in Line Broadening. II. Application to the Lyman Series of Hydrogen
}

\author{
Earl W. Smith, ${ }^{*}$ C. R. Vidal, ${ }^{*}$ and J Cooper** \\ Institute for Basic Standards, National Bureau of Standards, \\ Boulder, Colorado 80302
}

(February 28, 1969)

\begin{abstract}
The use of the classical path approximation in line broadening theory is illustrated in a development of the familiar impact and one-electron theories. The one-electron theory which is presented is an improved version of the usual one-electron theory: this improved version of the theory provides a consistent description of a line profile from the half width to the quasi-static wings. The validity criteria for the impact and one-electron theories are discussed in detail and a comparison of the theories is made with a view toward the development of a more general theory containing elements of both. To avoid unnecessary mathematical complications and to provide a more transparent comparison of the theories, the Stark broadening of the Lyman series in hydrogen is used as a specific example.
\end{abstract}

Key words: Classical path; impact theory; line broadening theory; one electron theory.

\section{Introduction}

In a previous paper $[1],{ }^{1}$ henceforth referred to as I, a general expression for the thermal average, eq (I-47), was derived using classical path methods. In this paper we will show how this expression may be used to derive the familiar results of the impact theories $[2,3,4]$ and the one-electron theories $[3,5]$. Possible improvements to these theories by means of a more general treatment of the thermal average, will also be discussed.

The perturber coordinates, $\mathbf{x}$ (eigenvectors of the perturber position operator) are integrated out of the expression for the thermal average when classical path methods are used (see eq $(\mathrm{I}-36)$ ). The only perturber variables which need to be considered in the expression for the thermal average, eq (I-47) are the coordinates $\mathbf{x}^{(i)}, \mathbf{v}^{(i)}$ of the perturber wave packets (which are viewed as classical particles). The superscript $(i)$ notation, which was used in I to distinguish between these variables, is no longer needed and, for convenience in notation, this superscript will not be used in this paper.

The thermal average will be evaluated by means of statistical techniques. In order to clearly illustrate these techniques and avoid unnecessary mathematical complications, we will consider the case of the Lyman lines emitted by hydrogen atoms in a plasma.

\footnotetext{
*Radio Standards Physies Division. Plasma Physies, Vational Bureau of Standards l.aboratories, Boulder. CiNo. 8030'2

** Joint Institute for Laboratory Astrophysics and Department of Physics and Astre. physies, I niversity of Colorado, Boulder, Colo. 80302.

1 rigures in brackets indicate the literature references at the end of this paper.
I
}

2. Preliminary Mathematics and Approximations

\subsection{The Line Shape in the Static Ion Approximation}

We will make the usual quasi-static approximation $[2,3]$ for the ions in the plasma. The electric field, $\mathscr{E}$, produced by these ions is regarded as being static during the time of interest, and this static field is used to define the $Z$ axis for the atom. $H_{a}$ is the Hamiltonian for an isolated hydrogen atom and, for convenience, the perturbation is taken to be a dipole interaction with the atomic dipole $-e \mathbf{R}$,

$$
\begin{gathered}
V(t)=e Z \mathscr{E}+V_{e}(\mathbf{R}, \mathbf{x}, \mathbf{v}, t) \\
V_{e}(\mathbf{R}, \mathbf{x}, \mathbf{v}, t)=e \mathbf{R} \cdot \mathscr{E}_{e}(\mathbf{x}, \mathbf{v}, t),
\end{gathered}
$$

where $Z$ denotes the $Z$ component of $\mathbf{R}$, and $\mathscr{E}_{e}(t)$ is the fluctuating electric field produced by the elec. trons. The classical variables $\mathbf{x}$ and $\mathbf{v}$ are $3 N$-vectors describing the $N$ electron perturbers (these were denoted by $\mathbf{x}^{(i)}$ and $\mathbf{v}^{(i)}$ in I). The average over ion fields is performed by means of the usual microfield average $[2,3,6]$. In this case, the complete line profile is given by

$$
\mathscr{I}(\omega)=\int \mathscr{P}(\mathscr{E}) I(\omega, \mathscr{E}) d \mathscr{E},
$$

where $\mathscr{P}(\mathscr{E})$ is the probability of finding an ion field of magnitude $\mathscr{E}$. The function $I(\omega, \mathscr{E})$ is the same as 
the function $I(\omega)$ discussed in $I$, except that we now explicitly indicate a dependence on the ion field $\mathscr{E}$. That is, we may regard the radiator as being an atom which is subjected to a static field $\mathscr{E}$ : the perturbers are electrons and the resulting line shape, for a given field $\mathscr{E}$, is $I(\omega, \mathscr{E})$ : the total line profile is then obtained by averaging $I(\omega, \mathscr{E})$ over all possible ion fields as shown in eq (3).

\subsection{The Time Development Operator in the No- Quenching Approximation}

The time development operator $T_{a}(t)$ (see eq (I-39)) is the solution of the differential equation

$$
\begin{gathered}
i \hbar \frac{\partial}{\partial t} T_{a}(t)=\left[H_{a}+e \mathscr{E} Z+V_{e}(t)\right] T_{a}(t) \\
T_{a}(0)=1 .
\end{gathered}
$$

In an interaction representation defined by

$$
T_{a}(t)=\exp \left(-i t H_{a} / \hbar\right) T_{a}^{\prime}(t),
$$

we have

$$
\begin{gathered}
i \hbar \frac{\partial}{\partial t} T_{a}^{\prime}(t)=\left[e \mathscr{E} Z^{\prime}(t)+V_{e}^{\prime}(t)\right] T_{a}^{\prime}(t) \\
Z^{\prime}(t)=\exp \left(i t H_{a} / \hbar\right) Z \exp \left(-i t H_{a} / \hbar\right) \\
V_{e}^{\prime}(t)=\exp \left(i t H_{a} / \hbar\right) V_{e}(t) \exp \left(-i t H_{a} / \hbar\right) .
\end{gathered}
$$

The formal solution of eq (6) may be given by

$T_{a \prime}^{\prime}(t)=\mathscr{O} \exp \left\{-(i / \hbar) \int_{0}^{t}\left[e \mathscr{E} Z^{\prime}\left(t^{\prime}\right)+V_{e}^{\prime}\left(t^{\prime}\right)\right] d t^{\prime}\right\}$

where $\mathcal{O}$ is the time ordering operator [7] which is required since $\left[e \mathscr{E} Z^{\prime}(t)+V_{e}^{\prime}(t)\right]$ does not commute with $\left[e \mathscr{E} Z^{\prime}\left(t^{\prime}\right)+V_{\rho}^{\prime}\left(t^{\prime}\right)\right]$ unless $t=t^{\prime}$ (cf. eq (6) of ref. [8]). Equation (9) is a very complicated expression and approximations must be employed to simplify the form of this operator. In this section we will consider the no-quenching approximation [9] which is frequently used for hydrogen lines.

To make an explicit statement of the no-quenching approximation, we must first introduce a complete set of $H_{11}$ eigenstates. The $H_{11}$ eigenvalues, $E_{\text {,., depend }}$ only on the principal quantum number $n$, hence we denote the $H_{n}$ eigenstates by $|n k\rangle$ where $h$ is some set of quantum numbers which index the degenerate states of the level $E_{\prime \prime}$. We are interested in the radiation produced when the atom makes a radiative transition from a group of initial states (degenerate states of

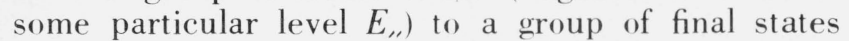
which have a lower energy. For the Lyman series in hydrogen, there is only one final state (i.e., the ground state neglecting spin) which we shall denote by $\mid 1>$; the frequency of the line of interest may then be denoted by $\omega_{, 11}=\left(E_{\prime \prime}-E_{1}\right) / \hbar$.

A radiationless transition which depopulates the initial level $E$ " may be said to quench the radiation near $\omega_{, 1}$. The probability for such an inelastic transition from an initial state $\mid$ eh $>$ to some other state $\left|r^{\prime} h^{\prime}\right\rangle$ (where $n \neq n^{\prime}$ ) is given by the square of $\left\langle\right.$ heti $\left.\left|T_{a}\right| n^{\prime} h^{\prime}\right\rangle$. The no-quenching approximation states that these "off-diagonal" (i.e., $n \neq \prime^{\prime}$ ) terms may be neglected (see eq (12) of ref. [9]). The justification for this approximation will be given in section 7; in this section we will simply use $<_{\text {rhti }}\left|T_{n}\right|$ rh $\left.^{\prime}\right\rangle=0$ to simplify the form of $T_{a}(t)$.

Since the "off-diagonal" matrix elements of $T_{11}(t)$ are assumed to be negligible, it is clear from eq (5), that the "off-diagonal" elements of $T_{" \prime}^{\prime}(t)$ must also be negligible. Noting that the $H_{a}$ eigenvalues depend only on $r$, the differential equation for $T_{a}^{\prime}(t)$, eq (6), becomes

$i \hbar \frac{\partial}{\partial t}\left\langle\right.$ nh $\left|T_{a}^{\prime}(t)\right|$ neti $\left.{ }^{\prime}\right\rangle$

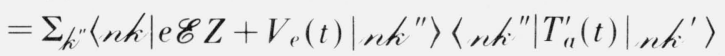

with the aid of eqs (7) and (8). If we define a projection operator $P$ which picks out the part of any operator which is diagonal in $n$

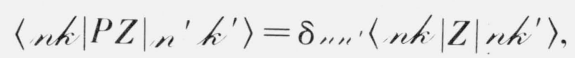

then eq (10) may be written

$$
i \hbar \frac{\partial}{\partial t} T^{\prime}(t)=\left[e \mathscr{E} P Z+P V_{e}(t)\right] T_{a}^{\prime}(t) .
$$

The appearance of the projection operator $P$ in the generator $\left[e \mathscr{E} P Z+P V_{e}(t)\right]$ indicates that $T_{a}^{\prime}(t)$ will be diagonal in $n$ as required. It is interesting to note that, while $V_{e}(t)$ commutes with $Z$ (they are functions of atomic position coordinates), the operators $P Z$ and $P V_{e}(t)$ will not commute in general. The formal solution of eq (11) will therefore be a time-ordered exponential similar to eq (9). We will define an operator $\mathscr{Z}(\mathscr{E})$ by

$$
\mathscr{Z}(\mathscr{E})=e \mathscr{E} P Z / \hbar
$$

and $T_{a}^{\prime}(t)$ will be written in an interaction representation defined by

where

$$
T_{a}^{\prime}(t)=\exp (-i t \mathscr{Z}) U_{a}(t)
$$

and

$$
i \hbar \frac{\partial}{\partial t} U_{a}(t)=\tilde{V}(t) U_{a}(t)
$$

$$
\tilde{V}(t)=\exp (\text { it } \mathscr{Z}) P V_{e}(t) \exp (-i t \mathscr{Z}) .
$$

With these new definitions, $T_{a}(t)$ may be written

$$
T_{a}(t)=\exp \left(-i t H_{a} / \hbar\right) \exp (-i t \mathscr{Z}) U_{a}(t),
$$


where $U_{a}(t)$ is the (time ordered) solution of eq (14):

$$
U_{a}(t)=\mathscr{O} \exp \left\{-(i / \hbar) \int_{0}^{t} \tilde{V}\left(t^{\prime}\right) d t^{\prime}\right\} .
$$

\subsection{The Line Shape for the Lyman Series}

The line shape function defined by eqs (I-7) and (I-11) involves a trace over atomic states hence this function will describe all spectral radiation emitted by the atom. Since we wish to study only one line (or a group of overlapping lines) at a time, we may pick out the relevant terms in this trace as discussed in section 7.2 of $\mathrm{I}$ (see eq (I-5l)). This selection may be accomplished by restricting the atomic dipole operator d to have matrix elements only between the initial and final states which contribute to the line, or lines, of interest (cf. eq (12) of ref. [9]).

For the Lyman lines in hydrogen there is only one final state (neglecting spin), which we have denoted by $|1\rangle$; the initial states for any given line are the $H_{a}$ eigenstates $|n k\rangle$ which correspond to the given

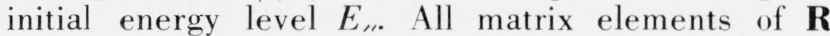
vanish in the ground state, $\langle 1|\mathbf{R}| 1\rangle=0$, hence we have $\left\langle 1\left|U_{a}\right| 1\right\rangle=1$ (this is why we have chosen the Lyman series as an example).

For convenience we will now specify the states $|n h\rangle$ to be the parabolic states which diagonalize both $[10,11] H_{a}$ and $P Z$. The diagonal elements of $Z$ will be denoted by $Z$, th and the diagonal elements of $H_{n}$ (the energy eigenvalues) have already been

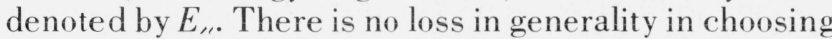
parabolic states because the line shape is defined in terms of a trace over atomic states and we are free to choose any complete set of basis states when evaluating a trace (i.e., a trace is invariant under a unitary transformation of the basis states). The atomic density matrix, $\rho^{\prime \prime}\left(H_{n}\right)$, is also diagonal in $|n h\rangle$ and its matrix elements are denoted by $\rho_{\prime \prime \prime}^{(\prime)}$.

Using eqs (I-7), (I-1]), (I-47) and defining a frequency variable $\Delta \omega=\left(\omega-\omega_{1,1}\right)$ for the particular line of interest, the line shape function $I(\omega, \mathscr{E})$ may be written in the form

$$
I(\omega, \mathscr{E})=\left(\rho_{\prime \prime \prime}^{(\prime \prime)} / \pi\right) \operatorname{Re} \Sigma_{\text {hith }}\left\langle\text { nht }^{\prime}|\mathbf{d}| 1\right\rangle \cdot\langle 1|\mathbf{d}| \text {, ehi }\rangle
$$

$$
\int_{0}^{\infty} \exp \left[i t\left(\Delta \omega-e \mathscr{E} Z_{, \ldots h} \mid \hbar\right)\right]\left\langle{ }_{n h}|F(t)| n h^{\prime}\right\rangle d t
$$

where

$$
\begin{gathered}
F(t)=Q^{-1} \iint \exp \left[-E_{\mu}(\mathbf{x}, \mathbf{v}) / k T\right] U_{l l}(\mathbf{R}, \mathbf{x}, \mathbf{v}, t) d \mathbf{x} \mathrm{d} \mathbf{v} . \\
E_{\mu}(\mathbf{x}, \mathbf{v})=\frac{1}{2} m v^{2}+V_{\mu}(\mathbf{x}) \\
Q=\iint \exp \left[-E_{\nu}(\mathbf{x}, \mathbf{v}) / k T\right] \mathrm{d} \mathbf{x} \mathrm{d} \mathbf{v}
\end{gathered}
$$

$E_{p}$ represents the energy of the $N$ electrons which are described by $\mathbf{x}=\left(\mathbf{x}_{1}, \mathbf{x}_{2}, \ldots, \mathbf{x}_{1}\right)$ and $\mathbf{v}=\left(\mathbf{v}_{1}, \mathbf{v}_{2}, \ldots\right.$. , $\mathbf{v})$, and $V_{\mu}(\mathbf{x})$ is the potential of interaction between electrons.

The thermal average is contained entirely in $F(t)$ and the remaining sections are devoted to the evaluation of this function. When $F(t)$ is known, the line shape is readily obtained from eqs (3) and (18).

\section{Further Simplification of the Time Development Operator}

\subsection{General Purpose}

Although the time development operator was considerably simplified by the no-quenching approximation, the resulting expression, eq (16), is still quite complicated and further simplifications are necessary in order to evaluate $F(t)$. To facilitate these simplifications we note that when we use the classical trajectories discussed in section 8.4 of $\mathrm{I}$, the interaction potential $V_{e}(t)$ in eq (2) may be written in the form

$$
\begin{gathered}
V_{e}(\mathbf{R}, \mathbf{x}, \mathbf{v}, t)=e \mathbf{R} \cdot \Sigma_{j} \mathscr{C}_{e}\left(\mathbf{x}_{j}, \mathbf{v}_{j}, t\right)=\Sigma_{j} V_{j}(t) \\
\mathscr{E}_{e}\left(\mathbf{x}_{j}, \mathbf{v}_{j}, t\right)=e\left(\mathbf{x}_{j}+\mathbf{v}_{j} t\right) / \mid \mathbf{x}_{j}+\mathbf{v}_{j} t^{3},
\end{gathered}
$$

where $\mathscr{E}_{e}\left(\mathbf{x}_{j}, \mathbf{v}_{j}, t\right)$ represents the electric field at the atom produced by the $j$ th perturber; $V_{j}(t)$ is simply a shorthand notation for $e \mathbf{R} \cdot \mathscr{E}_{e}\left(\mathbf{x}_{j}, \mathbf{v}_{j}, t\right)$, the interaction between the atom and the $j$ th perturber. The form of the potential, $\Sigma_{j} V_{j}(t)$, suggests that it may be possible to express $U_{a l}(t)$ in terms of a product of time development operators $U_{j}(t)$ for the individual electron-atom collisions. In section 3.4 it will be shown that such a product form is obtained without approximation if the time of interest is very short. For most cases however, the interaction $\tilde{V}(t)$ contains terms like

$$
\tilde{V}_{j}(t)=\exp (i t \mathscr{Z}) P V_{j}(t) \exp (-i t \mathscr{Z})
$$

which do not in general commute with one another, and this prevents us from obtaining the product form for $U_{l t}(t)$. Nevertheless, if the $V_{j}(t)$ do not overlap in time (or if this overlap is negligible) the ordered exponential in eq (17) may be expressed as an ordered product:

$$
\mathscr{O} \exp \left[-(i / \hbar) \int_{0}^{t} \tilde{V}\left(t^{\prime}\right) d t^{\prime}\right]
$$

$$
=\mathscr{O} \Pi_{j} \exp \left[-(i / \hbar) \int_{0}^{t} \tilde{V}_{j}\left(t^{\prime}\right) d t^{\prime}\right]
$$

where $\tilde{V}_{j}(t)$ is defined by

$$
\tilde{V}_{j}(t)=\exp (i t \mathscr{Z}) P V_{j}(t) \exp (-i t \mathscr{Z}) .
$$

(Equation (24) may be verified by comparing terms in the series expansions.) This result will be the basis of the following approximations. 


\subsection{The Impact Approximation}

The purpose of the impact approximation is to disentangle the overlapping collisions $[12,13]$ in $U_{a}(t)$ and permit the use of the product form stated in eq (24).

To do this, the impact approximation assumes that, for collisions which overlap in time, the average collision is weak enough that it may be described by a perturbation series in powers of $V_{j}(t)$. Collisions for which $(V \tau / \hbar)<1$ may be treated this way (cf. sec. 7 of I). Strong collisions, $(V \tau / \hbar)>1$, for which such an expansion is not practical, are assumed to be well separated in time so that two strong collisions never occur simultaneously. If a weak collision overlaps with a strong one, the weak collision is neglected and strong collisions are thus disentangled. The entanglement between overlapping weak collisions first appears in the second order terms in the series expansion of eq (24). In footnote 7 of ref. [13] it is stated the entanglement in the second order terms vanishes because the terms involving different electrons will average to zero (recall that $U_{a}(t)$ appears only under an average as stated in eq (19)). This result was obtained in ref. [13] by treating the electrons as statistically independent particles (using Debye shielded fields to approximate their correlations). Although we have not made such an assumption, we note from eq $(22)$ that the second order terms involve an electric field autocorrelation function $\left\langle\mathscr{E}_{e}(t) \mathscr{E}_{e}\left(t^{\prime}\right)\right\rangle$; and the use of statistically independent quasi-particles is known to be a good approximation in evaluating such functions. We may thus regard the impact approximation as being valid to second order for weak collisions.

Using eqs (17) and (24), we see that, in the impact approximation, $U_{a}(t)$ may be written in the form (cf. p. 497 of ref. [12])

$$
\begin{aligned}
& U_{a}(t)=\mathscr{O}_{0} \Pi_{j} U_{j}(t) \\
& U_{j}(t)=\mathscr{O} \exp \left[-(i / \hbar) \int_{0}^{t} \tilde{V}_{j}\left(t^{\prime}\right) d t^{\prime}\right],
\end{aligned}
$$

where $\mathscr{O}_{0}$ is a time ordering operator which keeps the collisions in chronological order; that is, the $U_{j}(t)$ which describes the first collision must be the first operator from the right in the product $\Pi_{j} U_{j}(t)$, and the second collision must be the second from the right, etc. To state the action of $\mathscr{O}_{0}$ more explicitly, we note that the variables $\mathbf{x}_{j}$ and $\mathbf{v}_{j}$ in $V_{j}(t)$ may be transformed into the collision variables $\rho_{0}, v_{0}, t_{0}$, etc., (see appendix) where $t_{0}$ is a reference time for the collision $\left(t_{0}\right.$ is the time of closest approach if the collision is completed). The operator $\mathscr{O}_{0}$ simply requires that $t_{0}$ for the first collision always be less than $t_{0}$ for the second collision, etc. (this ordering is necessary because the $\tilde{V}_{j}(t)$ for different collisions do not in general commute). It is important to note that, when the impact approximation is valid, $U_{j}(t)$ refers to a single collision which may be either weak or strong.

\subsection{The Line Wings}

In the wings of a line, the time of interest $1 / \Delta \omega$ (cf. eq $(I-52))$ is relatively short and it may be possible to neglect the exponentials, exp (it $\mathscr{Z})$, in $\tilde{V}(t)$. To show this, we write eq (14) in the form

$$
\begin{aligned}
& i \hbar \frac{\partial}{\partial t}\left\langle\text { nht }\left|U_{a}(t)\right| n h^{\prime}\right\rangle=\sum_{h^{\prime \prime}} \exp \left[\text { ite } \mathscr{E}\left(Z_{\text {, hi }}-Z_{\mu h^{\prime \prime}}\right) / \hbar\right] \\
& \left\langle\text { whi }\left|V_{e}(t)\right| \text { rehi" }\right\rangle\left\langle\text { nhi" }\left|U_{\text {"I }}(t)\right| \text { uhi }^{\prime}\right\rangle
\end{aligned}
$$

(recall that $U_{11}(t)$ is diagonal in $n$ ). If the time of interest, $(1 / \Delta \omega)$, is small enough that

$$
\Delta \omega>e \mathscr{E}_{\text {av }}\left(Z_{\mu h}-Z_{\mu h_{i}}\right) \hbar
$$

for all $h$ which pertain to the initial level, then the exponential in eq (28) may be neglected for $\mathscr{E} \leq \mathscr{E}_{\text {av }}$, where $\mathscr{E}_{\text {av }}$ denotes the average ion field strength. For $\mathscr{E}>\mathscr{C}_{\text {ar }}$, the electron broadening is negligible (as we will discuss in sec. 7), thus, if eq (29) is satisfied, we may neglect the exponentials involving $Z$. In this case, the solution of eq (28) is

$$
U_{a}(t)=O \exp \left\{-(i / \hbar) \int_{0}^{1} P V_{e}\left(t^{\prime}\right) d t^{\prime}\right\}
$$

and the time ordering is still necessary because $P V e(t)$ will not in general commute with $P V_{e}\left(t^{\prime}\right)$.

Using the impact approximation, $U_{11}(t)$ is again given by the product form stated in eq (26) and $U_{j}(t)$ is given by

$$
U_{j}(t)=O \exp \left\{-(i / \hbar) \int_{0}^{t} P V_{j}\left(t^{\prime}\right) d t^{\prime}\right\}
$$

Again the time ordering appears because $P V_{j}(t)$ does not commute with $P V_{j}\left(t^{\prime}\right)$.

For weak collisions the exponential is valid only to second order in $P V_{j}(t)$ (because of the impact approximation) and it can be shown that, to this order, the operator $O$ is unnecessary. To show this we note from eq (22) that $P V_{j}(t)$ may be written in the form

$$
P V_{j}(t)=e\left[P X \mathscr{C}_{x}(t)+P Y \mathscr{E}_{y}(t)+P Z \mathscr{C}_{z}(t)\right] .
$$

It is the orthogonal components of $P \mathbf{R}$ which do not commute; that is, $P X \mathscr{E}_{x}(t)$ commutes with $P X \mathscr{E}_{x}\left(t^{\prime}\right)$ but not with $P Y \mathscr{E}_{y}\left(t^{\prime}\right)$ or $P Z \mathscr{E}_{z}\left(t^{\prime}\right)$. To second order the terms which do not commute will contain factors like $\left\langle\mathscr{E}_{x}(t) \mathscr{E}_{y}\left(t^{\prime}\right)\right\rangle$ (recall that $U_{l l}(t)$ appears only under an average). For a spherically symmetric (about the atom) distribution of electrons these terms will average to zero. We may therefore drop the time ordering and write

$$
U_{j}(t)=\exp \left\{-(i / \hbar) \int_{0}^{t} P V_{j}\left(t^{\prime}\right) d t^{\prime}\right\}
$$

which is valid to second order in $P V_{j}(t)$ for the line wings (as specified by eq (29)). 
The above argument is not valid for strong collisions because the series expansion of $U_{j}$ is not applicable. We will nonetheless assume that eq (33) provides a good description of the influence of strong collisions: this approximation will be discussed in section 7 .

\subsection{The Quasi-Static Region}

In the far line wings where the time of interest, $1 / \Delta \omega$, is much less than the duration time $\tau$ for a collision,

$$
\tau \Delta \omega \gg 1,
$$

an interaction which would normally be regarded as dynamic (e.g., an electron-atom interaction) will take on a quasi-static aspect because the perturber motion during the time of interest is negligible. The transition from dynamic behavior in the line center to quasi-static behavior in the far wings is not properly described by current line shape theories because these theories approximate $U_{n}(t)$ by the first few terms in its series expansion and, as will be shown in section 7 , this approximation breaks down in the quasi-static wings. Nevertheless, it can be shown that $U_{a}(t)$ takes a relatively simple form in the quasi-static wings. In fact, the product form $\Pi_{j} U_{j}(t)$ may be obtained without approximation and no time ordering is necessary in the operators $U_{j}(t)$. To show this, we note that the time ordered exponential in eq (30) (which is valid in the wings) may be expressed as an exponential involving a series of commutators [14]:

$$
\begin{aligned}
& U_{a l}(t)=\exp \left\{-(i / \hbar) \int_{0}^{t} P V_{e}\left(t^{\prime}\right) d t^{\prime}\right. \\
& \left.\quad+\frac{1}{2}(i / \hbar)^{2} \int_{0}^{t} \int_{0}^{t^{\prime}}\left[P V_{e}\left(t^{\prime}\right), P V_{e}\left(t^{\prime \prime}\right)\right] d t^{\prime} d t^{\prime \prime}+\ldots\right\} .
\end{aligned}
$$

If the commutator is negligible, $P V_{e}(t)$ may be regarded as an operator which commutes with itself at different times; similarly we may regard $P V_{i}(t)$ and $P V_{j}\left(t^{\prime}\right)$ as commuting operators for all $i, j, t$, and $t^{\prime}$. In this case, $U_{a}(t)$ may be expressed in the product form $\Pi_{j} U_{j}(t)$ where $U_{j}(t)$ is given by eq (33).

To show when the commutator is negligible we expand $V_{e}(t)$ about $t=0$ (in the far wings $t$ is a small parameter). To lowest order the integral over $P V_{r}\left(t^{\prime}\right)$ is given by $t P V_{e}(0) / \hbar$ and the integral over $\left[P V_{e}\left(t^{\prime}\right)\right.$, $\left.P V_{e}\left(t^{\prime \prime}\right)\right]$ becomes $t^{3}\left[P V_{e}(0), P V_{e}^{\prime}(0)\right] / 6 \hbar^{2}$, where $V_{e}^{\prime}(0)$ denotes $\left(\partial V_{e}(t) / \partial t\right)$ at $t=0$. Since the magnitude of $\left[P V_{e}(0), P V_{e}^{\prime}(0)\right]$ is less than or equal to $P V_{\rho}(0) P V_{\rho}^{\prime}(0)$, we need only compare the magnitude of $\left(t^{2} P V_{e}^{\prime}(0) / 6 \hbar\right)$ with unity. To estimate this quantity we use eqs (22) and (23) and we obtain

$\left(\partial V_{e} / \partial t\right)=(\partial x / \partial t)\left(\partial V_{e} / \partial x\right) \simeq v_{0}\left(\partial V_{e} / \partial x\right) \simeq\left(v_{0} V_{e} / \rho_{0}\right)$,

where $\rho_{0}$ and $v_{0}$ denote the position and velocity at the time of closest approach (when $P V_{e}^{\prime}$ is largest). Using $\tau=\left(\rho_{0} / v_{0}\right)$ and $t=(1 / \Delta \omega)$, we obtain

$$
t^{2} P V_{e}^{\prime}(0) / 6 \hbar \simeq\left(V_{e} / \hbar \Delta \omega\right)(1 / \tau \Delta \omega) .
$$

For quasi-static interactions we have $V \simeq \hbar \Delta \omega$ (cf. p. 23 of ref. [4]) and $t^{2} P V_{\rho}^{\prime}(0) / 6 \hbar \simeq 1 / \tau \Delta \omega \ll 1$. Thus, if eq (34) is satisfied, the commutator in eq (35) is negligible and $U_{a}(t)$ may be expressed in the product form as stated.

\section{Series Expansions of $F(t)$}

In this section we will obtain series expansions for the function $F(t)$ defined by eq. (19). The various methods of handling these expansions give rise to different types of theories (e.g., the one-electron theory, impact theory, etc.).

The classical Hamiltonian $E_{p}(\mathbf{x}, \mathbf{v})$ appearing in $F(t)$ was given, in eq (20), by

$$
E_{p}(\mathbf{x}, \mathbf{v})=\sum_{j} m v_{j}^{2} / 2+V_{p}\left(\mathbf{x}_{1}, \ldots ., \mathbf{x}_{N}\right)
$$

where $\mathbf{x}_{j}$ and $\mathbf{v}_{j}$ refer to the $j$ th electron and $V_{p}(\mathbf{x})$ represents the electron-electron interaction. We may define position and velocity distribution functions $P(\mathbf{x})$ and $W(\mathbf{v})$ by

$$
\begin{gathered}
P(\mathbf{x})=\exp \left\{-V_{p}(\mathbf{x}) / k T\right\} / \int d \mathbf{x} \exp \left\{-V_{p}(\mathbf{x}) \downarrow k T\right\} \\
W\left(\mathbf{v}_{j}\right)=\exp \left\{-m v_{j}^{2} / 2 k T\right\} / \int d \mathbf{v}_{j} \exp \left\{-m v_{j}^{2} / 2 k T\right\} \\
W(\mathbf{v})=\prod_{j} W\left(\mathbf{v}_{j}\right),
\end{gathered}
$$

and these have the property that

$$
W(\mathbf{v}) P(\mathbf{x})=Q^{-1} \exp \left\{-E_{p}(\mathbf{x}, \mathbf{v}) / k T\right\}
$$

where $Q$ was defined by eq (21). Using the product form for $U_{a}(t)$ given in eq (26), we obtain

$$
F(t)=\mathscr{O}_{0} \int d \mathbf{x} P(\mathbf{x}) \int \Pi_{j} d \mathbf{v}_{j} W\left(\mathbf{v}_{j}\right) U_{j}(t) .
$$

Following the technique used by Baranger and Mozer [15], we define a function $\varphi\left(\mathbf{R}, \mathbf{x}_{j}, t\right)$ (which should not be confused with the wave functions $\varphi$ used in I) by

$1+\varphi\left(\mathbf{R}, \mathbf{x}_{j}, t\right)=\int d \mathbf{v}_{j} W\left(\mathbf{v}_{j}\right) U_{j}\left(\mathbf{R}, \mathbf{x}_{j}, \mathbf{v}_{j}, t\right)$.

Using $\varphi(j)$ as a shorthand notation for $\varphi\left(\mathbf{R}, \mathbf{x}_{j}, t\right)$ we have

$$
\Pi_{j}[1+\varphi(j)]=1+\sum_{j} \varphi(j)+\sum_{j<k} \varphi(j) \varphi(k)+\ldots .
$$

Substituting eqs (42) and (43) into (41) we obtain the series

$$
F(t)=\mathscr{O}_{0}\left[1+\sum_{M=1}^{N} F_{M}(t)\right]
$$

where

$F_{M}(t)=\sum_{j_{1}<j_{2}<\ldots<j_{M}} \int \varphi\left(j_{1}\right) \ldots \varphi\left(j_{M}\right) P(\mathbf{x}) d \mathbf{x}$. 
Since $P\left(\mathbf{x}_{1}, \ldots, \mathbf{x}_{N}\right)$ is invariant under an interchange of particle coordinates we have

$F_{M}(t)=[N ! / M !(N-M) !]$

$\int \ldots \int \varphi(1) \ldots \varphi(M) P\left(\mathbf{x}_{1}, \ldots, \mathbf{x}_{N}\right) d \mathbf{x}_{1} \ldots d \mathbf{x}_{N}$

$$
=[N ! / M !(N-M) !]
$$

$\int \ldots \int \varphi(1) \ldots \varphi(M) P_{M}\left(\mathbf{x}_{1}, \ldots, \mathbf{x}_{M}\right) d \mathbf{x}_{1} \ldots d \mathbf{x}_{M}$,

where the reduced distribution function $P_{M}\left(\mathbf{x}_{1}, \ldots\right.$., $\left.\mathbf{x}_{M}\right)$ is defined by

$$
\begin{aligned}
& P_{M}\left(\mathbf{x}_{1}, \ldots, \mathbf{x}_{M}\right)=\int \ldots \int P\left(\mathbf{x}_{1}, \ldots,\right. \\
&\left.\mathbf{x}_{M}, \mathbf{x}_{M+1}, \ldots, \mathbf{x}_{N}\right) d \mathbf{x}_{M+1} \ldots . d \mathbf{x}_{N} .
\end{aligned}
$$

$P_{M}\left(\mathbf{x}_{1}, \ldots, \mathbf{x}_{M}\right) d \mathbf{x}_{1} \ldots d \mathbf{x}_{M}$ represents the probability of finding the $M$ particles labeled 1 through $M$ in the volumes $d \mathbf{x}_{1}, \ldots d \mathbf{x}_{M}$ at the points $\mathbf{x}_{1}, \ldots, \mathbf{x}_{M}$ (for a further discussion see ref. [16]). The combinatorial factor in eq (46) represents the number of ways for choosing $M$ electrons from the perturbing gas of $N$ identical electrons.

The reduced distribution functions $P_{M}\left(\mathbf{x}_{1}, \ldots, \mathbf{x}_{M}\right)$ may be expanded in an Ursell expansion [17], the first three terms of which are given by

$$
\begin{aligned}
& \mathscr{V} P_{1}\left(\mathbf{x}_{1}\right)=g_{1}\left(\mathbf{x}_{1}\right), \\
& \qquad \mathscr{V}^{2} P_{2}\left(\mathbf{x}_{1}, \mathbf{x}_{2}\right)=g_{1}\left(\mathbf{x}_{1}\right) g_{1}\left(\mathbf{x}_{2}\right)+g_{2}\left(\mathbf{x}_{1}, \mathbf{x}_{2}\right), \\
& \quad \mathscr{V}^{3} P_{3}\left(\mathbf{x}_{1}, \mathbf{x}_{2}, \mathbf{x}_{3}\right)=g_{1}\left(\mathbf{x}_{1}\right) g_{1}\left(\mathbf{x}_{2}\right) g_{1}\left(\mathbf{x}_{3}\right) \\
& +g_{1}\left(\mathbf{x}_{1}\right) g_{2}\left(\mathbf{x}_{2}, \mathbf{x}_{3}\right)+g_{1}\left(\mathbf{x}_{2}\right) g_{2}\left(\mathbf{x}_{1}, \mathbf{x}_{3}\right) \\
& \quad+g_{1}\left(\mathbf{x}_{3}\right) g_{2}\left(\mathbf{x}_{1}, \mathbf{x}_{2}\right)+g_{3}\left(\mathbf{x}_{1}, \mathbf{x}_{2}, \mathbf{x}_{3}\right),
\end{aligned}
$$

where $\mathscr{V}$ is the volume of the system; the general term in the Ursell expansion is given by eq (9) of ref. [17]. The function $g_{1} / \mathscr{V}$ is the one-body probability function; $g_{1}\left(\mathbf{x}_{1}\right) g_{1}\left(\mathbf{x}_{2}\right) / \mathscr{V}^{2}$ would be the two-body probainility function if there were no correlation between particles. Since there is a correlation between the particles at $\mathbf{x}_{1}$ and $\mathbf{x}_{2}$, this correlation is represented by the twobody correlation function $g_{2}\left(\mathbf{x}_{1}, \mathbf{x}_{2}\right)$. Similarly $P_{3}$ is expressed by a group of terms which contain no more than two-body correlations plus $g_{3}\left(\mathbf{x}_{1}, \mathbf{x}_{2}, \mathbf{x}_{3}\right)$, the three-body correlation function. The general term in the Ursell expansion expresses $P_{M}\left(\mathbf{x}_{1}, \ldots, \mathbf{x}_{M}\right)$ as a sum of terms which contain no more than $(M-1)$-body correlations plus $g_{M}\left(\mathbf{x}_{1}, \ldots, \mathbf{x}_{M}\right)$, the $M$-body correlation function. Following Baranger and Mozer [15] we define a function

$$
\begin{array}{r}
h_{M}(t)=\int \varphi(1) \ldots \varphi(M) g_{M}\left(\mathbf{x}_{1}, \ldots .\right. \\
\left.\mathbf{x}_{M}\right) d \mathbf{x}_{1} \ldots d \mathbf{x}_{M},
\end{array}
$$

and, after considerable algebra, it is found that $F(t)$, as given by eqs (41) and (43), can be expanded in terms of the $g_{M}$ and then resummed to yield

$$
F(t)=\mathscr{O}_{0} \exp \left\{\sum_{M=1}^{\infty}\left(n^{M} / M !\right) h_{M}(t)\right\} .
$$

In the derivation of eq (50) it was necessary to let $N$ and $\mathscr{V}$ become infinite in such a way that the density $n=N / \mathscr{V}$ remained constant. In this new series for $F(t)$, each successive term represents a higher order correlation effect.

We now have two entirely different expressions (eqs (44) and (50)) for $F(t)$. To determine the utility of these expressions, we will briefly outline the physical significance of the terms in the series expansions involved. To understand the physical significance of $F(t)$ itself, we note that, except for multiplicative constants (e.g., d matrix elements), $F(t)$ is the same as the autocorrelation function $C(t)$ discussed in section 7 of $\mathrm{I}$.

The $F_{1}(t)$ term in eq (44) describes the electronatom interactions (collisions) as though they are completely independent of one another. From eqs. (42) and (46) we see that $F_{1}(t)$ is given by a sum of terms, each of which contains only one $U_{j}$ operator, thus, to this order the effects of the interactions are simply additive. The terms $F_{2}, \ldots, F_{N}$ account for the fact that the effect on $F(t)$ of one interaction may be influenced by other interactions. When this influence is taken into account, the effects of the collisions are no longer simply additive. This influence of one interaction on another comes about in basically two ways. The most obvious influence is due to the correlation between the perturbing electrons which are described by the correlation functions $g_{M}$ for $M \geqslant 2$. However, even when these correlations are ignored (by setting $g_{M}=0$ for $M \geqslant 2$ ), the $F_{2}, \ldots, F_{M}$ terms do not vanish and it is still possible for the outcome of one collision to be influenced by other collisions. This remaining influence comes about when a collision, or a series of collisions, produces a large change in the state of the radiator thereby reducing the autocorrelation function $F(t)$. If the state of the radiator is thus materially altered from its original value, the effect of subsequent collisions will be correspondingly modified. For example, there is a high probability that a strong collision will cause an inelastic transition between states having different principal quantum numbers and it is obvious that such a transition cannot be neglected when calculating the effect of the next collision. Since it takes several weak collisions to build up such an influence, we expect that $F_{1}(t)$ will provide a good approximation to $F(t)$ for times which are shorter than the mean free time between strong collisions (for those cases where a strong collision does occur during this time, the effects of subsequent weak collisions are negligible in comparison). For longer times of interest it is necessary to consider all of the terms $F_{1}, \ldots, F_{N}$, and in this case eq $(50)$ is more appropriate than eq (44). 
In eq $(50)$, the $h_{1}(t)$ term describes the electronatom interactions as though the position of an electron is not influenced by the presence of the other electrons. That is, the electron-electron correlations are neglected in this term (although the remaining influence of one collision on the effects of subsequent collisions is properly included). The $h_{2}$ term accounts for pair correlations between electrons, and subsequent terms describe higher order correlations. Since it is usually not necessary to consider more than pair correlations, it would seem that eq (50) is much more useful than eq (44). This would indeed be the case if we could always make calculations based on eq (50); as we will show in the following sections, the calculations based on eq (50) become quite difficult for short times of interest and in this case eq (44) is of more practical value.

As a further comparison of eq (44) and (50), it is interesting to note that, in the microfield theory [15], the approximation $F \simeq \exp \left(n h_{1}\right)$ gives rise to the Holtsmark distribution function (no correlation approximation) whereas the approximation $F \simeq 1+F_{1}$ gives the asymptotic Holtsmark function (or nearest neighbor approximation). This indicates that the approximation $F(t) \simeq \mathscr{O}_{0}\left[1+F_{1}(t)\right]$ is just the small $t$ limit of $F(t) \simeq \mathscr{O}_{0}$ exp $\left\{n h_{1}(t)\right\}$. To verify that this is the case, we note from eqs (42) and (49) that as $t \rightarrow 0, h_{1}(t) \rightarrow 0$ and $\exp \left\{n h_{1}(t)\right\} \rightarrow 1+n h_{1}(t)$; substituting the functions [16] $P_{1}\left(x_{1}\right)=n / N$ and $g_{1}\left(x_{1}\right)=1$ into eqs (45) and (49) we see that $F_{1}(t)=n h_{1}(t)$, thus $1+F_{1}(t)$ is indeed the small $t$ limit of $\exp \left\{n h_{1}(t)\right\}$.

\section{The One-Electron Theory}

The one-electron theory is designed to provide a description of the wings of a line profile. The version of this theory presented in this section will be shown (in sec. 7) to provide a consistent description of a line profile from the half width to the quasi-static wings. This theory is based on the approximation $F(t) \simeq \mathscr{O}_{0}\left[1+F_{1}(t)\right]$ which is valid for small times of interest or large $\Delta \omega$. Since $F_{1}(t)$ is given by a sum of terms each of which contains a single $U_{j}(t)$ operator (rather than products of the $U_{j}$ ), the chronological ordering operator $\mathcal{O}_{0}$ will have no effect on $F_{1}$. Using [16] $P_{1}\left(x_{1}\right)=n / N$ in eq (45), we obtain

$$
F(t)=1+n \int \varphi\left(\mathbf{R}, \mathbf{x}_{1}, t\right) d \mathbf{x}_{1} .
$$

Notice that this expression involves the average of the time development operator for a single collision; this is the origin of the name "one-electron" theory.
The first term in $F(t)$ (i.e., the constant 1) will give rise to a delta function, $\delta\left(\Delta \omega-e \mathscr{E} Z_{n \hbar} \hbar\right)$, when it is substituted into eq (18). Performing the ion microfield average, as required by eq (3), it is clear that the contribution to $\mathscr{I}(\omega)$ resulting from this term will be proportional to $\mathscr{P}\left(\hbar \Delta \omega / e Z_{n}\right)$. This represents the line shape which would be produced by the ions alone.

The influence of the electrons (as well as some electron-ion coupling) is contained in the second term in eq (51):

$$
F_{1}(t)=n \int \varphi\left(\mathbf{R}, \mathbf{x}_{1}, t\right) d \mathbf{x}_{1} .
$$

Using eqs (33) (which is valid only in the line wings) and (42), and noting that $\int W\left(v_{1}\right) d \mathbf{v}_{1}=1$, we obtain

$$
\begin{aligned}
& F_{1}(t)=n \int d \mathbf{v}_{1} W\left(v_{1}\right) \int d \mathbf{x}_{1} \\
& {\left[\exp \left\{-(i / \hbar) \int_{0}^{t} P V_{1}\left(\mathbf{R}, \mathbf{x}_{1}+\mathbf{v}_{1} t^{\prime}\right) d t^{\prime}\right\}-1\right] .}
\end{aligned}
$$

As discussed in the appendix, we may transform to the more familiar collision variables $\left(\boldsymbol{\rho}_{0}, \mathbf{v}_{0}, t_{0}\right)$ where $\boldsymbol{\rho}_{0}$ and $\mathbf{v}_{0}$ denote the impact parameter and electron velocity while $t_{0}$ is some reference time in the collision (the time of closest approach in a completed collision). In terms of these variables we have

$$
\begin{aligned}
& F_{1}(t)=n \int d \Omega \int_{0}^{\infty} v 3 W\left(v_{0}\right) d v_{0} \int_{0}^{\infty} \rho_{0} d \rho_{0} \int_{-\infty}^{+\infty} d t_{0} \\
& {\left[\exp \left\{-(i / \hbar) \int_{0}^{t} P V_{1}\left(\mathbf{R}, \boldsymbol{\rho}_{0}, \mathbf{v}_{0}, t^{\prime}+t_{0}\right) d t^{\prime}\right\}-1\right]}
\end{aligned}
$$

where

$$
\begin{aligned}
V_{1}\left(\mathbf{R}, \boldsymbol{\rho}_{0}, \mathbf{v}_{0}, t^{\prime}+t_{0}\right)=e^{2} \mathbf{R} \\
\cdot\left[\boldsymbol{\rho}_{0}+\mathbf{v}_{0}\left(t^{\prime}+t_{0}\right)\right] /\left[\rho_{0}^{2}+v_{0}^{2}\left(t^{\prime}+t_{0}\right)^{2}\right]^{3 / 2}
\end{aligned}
$$

The Euler angles $\Omega$ denote the orientation of the "collision axes" relative to the direction of $\mathbf{R}$, the atomic dipole. We may therefore interpret the integral over $\Omega$ as an average over all possible orientations of the electron trajectory [5] relative to the direction of $\mathbf{R}$.

To make full use of the collision axes, we take the matrix elements of $F_{1}(t)$ as required by eq (18). These matrix elements are given by

$$
\begin{aligned}
&\left\langle n h\left|F_{1}(t)\right| n h^{\prime}\right\rangle=n \int d \Omega \int_{0}^{\infty} v_{0}^{3} W\left(v_{0}\right) d v_{0} \int_{0}^{\infty} \rho_{0} d \rho_{0} \int_{-\infty}^{\infty} d t_{0} \\
&\left\langle n \hbar\left|\left[\exp \left\{-(i / \hbar) \int_{0}^{t} P V_{1}\left(\mathbf{R}, \boldsymbol{\rho}_{0}, \mathbf{v}_{0}, t^{\prime}+t_{0}\right) d t^{\prime}\right\}-1\right]\right| n h^{\prime}\right\rangle .
\end{aligned}
$$


We may now perform a rotation of the "atomic axes," through the Euler angles $\Omega$, so that $\mathbf{R}$ will point in the same direction as $\boldsymbol{\rho}_{0}$, and $\mathbf{v}_{0}$ defines the $x$ direction. The rotated wave functions will be given by [18] $\mathscr{D}(\Omega)|n h\rangle$, where $\mathscr{D}(\Omega)$ is a rotation operator. The interaction potential referred to collision axes is

$$
\begin{aligned}
& V_{c}\left(X, Z, \rho_{0}, v_{0}, t^{\prime}+t_{0}\right) \\
& \quad=e^{2}\left[Z \rho_{0}+X v_{0}\left(t^{\prime}+t_{0}\right)\right] /\left[\rho_{0}^{2}+v_{0}^{2}\left(t^{\prime}+t_{0}\right)^{2}\right]^{3 / 2},
\end{aligned}
$$

where $X$ and $Z$ denote the $x$ and $z$ components of $\mathbf{R}$, and we have the identity

$$
\begin{aligned}
\left\langle\text { uh }\left|\left[\exp \left\{-(i / \hbar) \int_{0}^{t} P V_{1}\left(\mathbf{R}, \rho_{0}, v_{0}, t^{\prime}+t_{0}\right) d t^{\prime}\right\}-1\right]\right| n \hbar^{\prime}\right\rangle \\
=\left\langle n h\left|\mathscr{D}^{-1}(\Omega)\left[\exp \left\{-(i / \hbar) \int_{0}^{t} P V_{c}\left(X, Z, \rho_{0}, v_{0}, t^{\prime}+t_{0}\right) d t^{\prime}\right\}-1\right] \mathscr{D}(\Omega)\right| n h^{\prime}\right\rangle .
\end{aligned}
$$

Substituting this identity into eq (56), we obtain

$$
\left\langle n k\left|F_{1}(t)\right| n k^{\prime}\right\rangle=\int\left\langle n k\left|\mathscr{D}^{-1}(\Omega) F_{1}^{c}(t) \mathscr{D}(\Omega)\right| n k^{\prime}\right\rangle d \Omega \text {, }
$$

where

$$
\begin{aligned}
& F_{\mathrm{i}}^{\mathrm{c}}(t) \equiv n \int_{0}^{\infty} v_{3}^{3} W\left(v_{0}\right) d v_{0} \int_{0}^{\infty} \rho_{0} d \rho_{0} \int_{-\infty}^{\infty} d t_{0} \\
& \quad\left[\exp \left\{-(i / \hbar) \int_{0}^{t} P V_{c}\left(X, Z, \rho_{0}, v_{0}, t^{\prime}+t_{0}\right) d t^{\prime}\right\}-1\right] .
\end{aligned}
$$

The average over $\Omega$ in eq (59) may be readily performed by transforming to spherical wave functions [19] and using the properties of $\mathscr{D}(\Omega)$ as given by Edmonds [18]. The function $F_{\mathrm{i}}(t)$ may be evaluated by numerical methods, and the line shape is then obtained by substituting the result into eq (18) and performing the transform. Further details of such a calculation together with numerical results will be reported in a future paper.

\section{The Impact Theory}

To obtain the results of the impact theories we use the $U_{j}(t)$ which were obtained by the impact approximation, eq (27), and the approximate calculation of $F(t)$ is based on its expansion in terms of correlation functions, eq (50). For most plasma broadening problems one is interested in a temperature-density range where three-body correlations are negligible, hence it is necessary to consider only $h_{1}(t)$ (no correlations) and $h_{2}(t)$ (two-body correlations). In the impact theories, electron correlations are not treated with correlation functions (such as $g_{2}\left(\mathbf{x}_{1}, \mathbf{x}_{2}\right)$ which appears in $\left.h_{2}(t)\right)$. Instead, in these theories the electron correlations are approximated by impact parameter cutoffs. Shielded field arguments are used to derive a cutoff of approximately $\lambda_{l}$, the Debye length, in the range of the electron-atom interaction [20|. and the frequency ciependent Lewis cutoff [21] $\left(v_{\mathrm{av}} / \Delta \omega\right)$ is used in place of $\lambda_{l}$, when $\left(v_{\text {av }} / \Delta \omega\right)<\lambda_{l}$ (this will be discussed in sec. 7). A similar cutoff behavior has also been obtained, by means of correlation functions, within the framework of a quantum mechanical theory [22]. However a more accurate treatment of the electron correlations [23] has shown that, while the cutoff at $\left(v_{\mathrm{av}} / \Delta \omega\right)$ is valid for $\Delta \omega>\omega_{p}$, the cutoffs in the line center are in error. The cutoff at the Debye length (or at $1.1 \lambda_{l}$ ) in some theories) should be multiplied by $(2.718)^{-1 / 2} \simeq 0.6$ resulting in a change on the order of 20 percent in the broadening function $\Phi_{a b}$ used by Griem et al. $[2,9,13,20]$. For $\Delta \omega \simeq \omega_{p}$, there is a possibility of further corrections due to dynamic correlation effects.

To derive the results obtained by the impact theory, we will assume that the effect of electron correlations may be represented by some correctly chosen impact parameter cutoffs. We thus need to consider only the function $h_{1}(t)$ as given by eq (49):

$$
F(t) \simeq \mathscr{O}_{0} \exp \left\{n h_{1}(t)\right\}
$$

For a fluid [16] such as a plasma, $g_{1}\left(\mathbf{x}_{1}\right)=1$ and $h_{1}$ is given by

$$
h_{1}(t)=\iint d \mathbf{x}_{1} d \mathbf{v}_{1} W\left(\mathbf{v}_{1}\right)\left[U_{1}\left(\mathbf{R}, \mathbf{x}_{1}, \mathbf{v}_{1}, t\right)-1\right] .
$$

We note in passing that if the exponential in eq (61) is expanded, the first two terms in the series are identical with the approximate expression for $F(t)$, eq (51), which is used in the one-electron theory. A further discussion of this correspondence is given in the following sections.

Using eqs (25) and (27), $h_{1}(t)$ is given by

$$
\begin{gathered}
h_{1}(t)=\iint d \mathbf{x}_{1} d \mathbf{v}_{1} W\left(\mathbf{v}_{1}\right)\left[\mathscr { O } \operatorname { e x p } \left\{-(i / \hbar) \int_{0}^{t} e^{i t^{\prime} \mathscr{L}}\right.\right. \\
\left.\left.P V_{1}\left(\mathbf{R}, \mathbf{x}_{1}, \mathbf{v}_{1}, t^{\prime}\right) e^{-i t^{\prime} \mathscr{Z}} d t^{\prime}\right\}-1\right] .
\end{gathered}
$$

Transforming to collision variables (see appendix) and denoting the average over $\rho_{0}, v_{0}$, and $\Omega$ by a subscript av, this becomes 


$$
h_{1}(t)=\int_{-\infty}^{\infty} d t_{0}\left[\mathcal{O} \exp \left\{-(i / \hbar) \int_{0}^{t} e^{i t^{\prime} \mathscr{X}} P V_{1}\left(t^{\prime}+t_{0}\right) e^{-i t^{\prime} \mathscr{X}} d t^{\prime}\right\}-1\right]_{\mathrm{av}} .
$$

Changing variables from $t^{\prime}$ to $s=t^{\prime}+t_{0}$ and using the identity $\exp \left[e^{-A} B_{e}^{A}\right]=e^{-A} e^{B} e^{A}$, we obtain

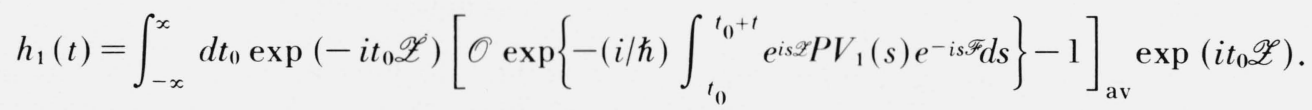

We assume that a collision time $\tau$ exists such that $V_{1}(s)=0$ unless $0 \leqslant s \leqslant \tau$. The completed collision assumption, which is an important part of most impact theories, states that if a collision occurs, it can be completed. That is, if some value of $s$ in the interval $\left[t_{0}, t_{0}+t\right]$ satisfies $0 \leqslant s \leqslant \tau$, then the completed collision assumption states that the collision should be completed in that interval, i.e., $t_{0} \leqslant 0 \leqslant s \leqslant \tau \leqslant\left(t_{0}+t\right)$. Using this restriction on $t_{0}$ and extending the limits $\left(t_{0}, t+t_{0}\right)$ on the $s$ integral to $(-\infty,+\infty)$ (since $V_{1}(s)=0$ outside the range $0 \leqslant s \leqslant \tau$ anyway), eq (65) becomes

$$
\begin{aligned}
& h_{1}(t)=\int_{-(t-\tau)}^{0} d t_{0} \exp \left(-i t_{0} \mathscr{Z}\right)[S-1]_{\mathrm{av}} \exp \left(i t_{0} \mathscr{Z}\right) \\
& =\int_{0}^{(t-\tau)} d t_{0} \exp \left(i t_{0} \mathscr{Z}\right)[S-1]_{\mathrm{av}} \exp \left(-i t_{0} \mathscr{Z}\right)
\end{aligned}
$$

where $S$ is an $S$-matrix for a single collision (weak or strong) defined by

$$
S=\mathcal{O} \exp \left\{-(i / \hbar) \int_{-\infty}^{x} e^{i s \mathscr{Y}} P V_{1}(s) e^{-i s \mathscr{X}} d s\right\}
$$

The completed collision assumption will provide a good approximation to $h_{1}(t)$ since $\tau$ is usually very small (i.e., $t \gg \tau$ ), however, if $t<\tau$, the collision cannot be completed and this approximation breaks down. Using the time of interest $1 / \Delta \omega$ from eq (I-52), we may state that the completed collision assumption will be valid when

$$
\tau \Delta \omega \ll 1,
$$

where $\tau$ is some "representative" collision time [3]. This inequality is sometimes used as the validity criterion for the impact approximation [3]; this point will be discussed further in section 7 .

Substituting eq (66) in eq (61) and using the operator identity

$$
\mathcal{O}_{0} \exp \left[\int_{0}^{t} \exp \left(-t_{0} A\right) B \exp \left(t_{0} A\right) d t_{0}\right]=e^{-t \cdot e^{t(A+B)}}
$$

we obtain

$$
\begin{aligned}
F(t) & =e^{i(t-\tau) \mathscr{L}} \exp \left[-i(t-\tau) \mathscr{Z}+(t-\tau) \Phi_{a}\right] \\
& =e^{i(t-7) \mathscr{X}} \exp \left[+i \tau \mathscr{Z}-\tau \Phi_{l}\right] \exp \left[-i t \mathscr{Z}+t \Phi_{l}\right],
\end{aligned}
$$

where (cf. eq (42) of ref. [3] or eq (28) of ref. [12])

If we use

$$
\Phi_{a}=n[S-1]_{\mathrm{av}} .
$$

$$
\tau \Phi_{a} \ll 1
$$

which is simply a statement that the impact approximation is valid (as we will show in sec. 7), then eq (70) becomes (cf. eq (39) in ref. [3] or eq (30) of ref. [12])

$$
F(t)=e^{i t \mathscr{Z}} \exp \left[-i t \mathscr{Z}+t \Phi_{a}\right] .
$$

Substituting this $F(t)$ into eq (18), we obtain

$$
I(\omega, \mathscr{E})=\left(\rho_{\prime \prime}^{(\prime \prime)} \mid \pi\right) \operatorname{Re} \sum_{h h^{\prime}}\langle n h|\mathbf{d}| 1\rangle \cdot\left\langle 1|\mathbf{d}| n h^{\prime}\right\rangle
$$

$\int_{0}^{\infty}\left\langle n \hbar\left|\exp \left[i(\Delta \omega-e \mathscr{E} P Z \mid \hbar) t+t \Phi_{l}\right]\right| n \hbar^{\prime}\right\rangle d t$,

Comparing with eqs (9) and (32) of ref. [13], we see that this result is formally identical with the results obtained by the impact theory for the Lyman series.

It is important to note that, in most calculations of hydrogen line broadening, the exponentials exp $($ is $\mathscr{Z}$ ) in the integrand of the $S$-matrix (eq 67)) are either ignored or approximated by a cutoff [24]. To show how this cutoff is obtained, we note that when $e \mathscr{E} \tau\left(Z, \ldots-Z, L_{i}\right) / \hbar \ll 1$ the exponentials may be replaced by unity. When $e \mathscr{E} \tau\left(Z, \ldots, Z, k_{1}\right) / \hbar \gg 1$, the exponentials oscillate rapidly and there will be only a negligible contribution from this region of the integral. Defining a frequency

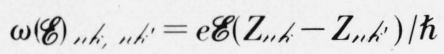

and noting that $\tau=\rho_{0} / v_{0}$, we define an impact param-

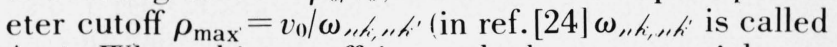
$\left.\Delta \omega_{s}\right)$. When this cutoff is used, the exponentials exp (is $\mathscr{Z}$ ) in eq (67) are replaced by unity; this cutoff reduces the integrand to zero when $\tau \omega_{\text {, }} k_{\text {, }}, t_{i}>1$, thus approximating the effect of the rapidly oscillating exponentials, and when $\tau \omega_{\text {, }}, h_{i}<1$ the exponentials are effectively replaced by unity. This cutoff is used simply as a method of simplifying the numerical calculations and it could be corrected without any modification of the theory itself. 


\section{Discussion of Approximations}

\subsection{General}

In the preceding sections we have derived the familiar results of the impact and one-electron theories using the classical path formalism developed in I. The advantage of these theories lies in their simple classical description of the perturber dynamics; their disadvantage with respect to more recent quantum theories $[6,22,25]$, is in the large number of approximations which are necessary to achieve this classical description. Since an understanding of these approximations plays an important role in the application and improvement of classical path theories, these approximations will be discussed in some detail in this section.

\subsection{Preliminary Approximations}

In our treatment of both the impact and the oneelectron theories we have used a dipole approximation to the perturbation potential, a static treatment of the ion field, and the no-quenching approximation. It should also be mentioned that we have not considered any perturbation of the final state in the radiative transition because there is no linear Stark effect in the ground state of hydrogen and any higher order ground state perturbations (e.g., quadratic Stark effect) are unimportant [26]. For lines other than those in the Lyman series, lower state perturbations may have to be considered.

To show that the static approximation provides a good description of the ions, we consider the change $\delta \mathscr{E}$ in an electric field $\mathscr{E}=e / r^{2}$ during a time $\delta t$. Using $\delta \mathscr{E} \simeq \delta t(\partial \mathscr{E} / \partial t) \simeq v \delta t(\partial \mathscr{E} / \partial r) \simeq(v \mathscr{E} \delta t / r)$, we see that $(\delta \mathscr{E} / \mathscr{E})$ may be estimated by $(\delta t / \tau)$ where $\tau$ is the duration of a collision. If $\delta t$ is replaced by the time of interest $(1 / \Delta \omega)$, we see that $(\delta \mathscr{E} / \mathscr{E})$ will be negligible when

$$
\tau \Delta \omega \gg 1,
$$

as mentioned earlier. If a perturber is quasi-static it produces a Stark splitting $\hbar \Delta \omega$ given by $\hbar \Delta \omega=V$ (see p. 23 of ref. [4]). Using $V \simeq Z e^{2} R_{0} / \rho_{0}^{2}$ for an ion of charge $Z e$, this means that $\rho_{0}^{2} \simeq Z e^{2} R_{0} / \hbar \Delta \omega$. Since the velocity distribution is sharply peaked about $v_{\text {av }}$, we estimate $\tau$ by $\left(\rho_{0} / v_{\text {av }}\right)$ and eq (76) becomes

$$
\Delta \omega \gg \Delta \omega_{c}=\left(\hbar v_{\mathrm{av}}^{2} / e^{2} R_{0}\right) .
$$

The critical frequency $\Delta \omega_{c}$ is sometimes referred to as the Weisskopf frequency. Since $\Delta \omega_{c}$ for ions lies almost at the center of most lines, the static ion approximation is usually justified over most of the line profile (however see also ref. [27]). In section 3.E of ref. [6], it is shown that the effect of a static ion field may be described by the microfield average stated in eq (3).

In the no-quenching approximation it was assumed that the $T_{a}^{\prime}(t)$ matrix elements between states having different principal quantum numbers could be neglected when calculating $C(t)$. These "off-diagonal" matrix elements represent collision induced transitions which transfer the excitation of the atom from one energy level to another. In section 7.2 of I, it was shown that collision induced transitions between excited states correspond to switching the mode of oscillation in a classical oscillator. It was also shown that this mode switching effect could be neglected if the energy separation of the excited states is much larger than the halfwidth of the lines being studied. In hydrogen, states having the same $n$ are degenerate (or nearly degenerate in the presence of an ion field) and collision induced transitions between these states are very important; this is the reason for regarding all states with the same $n$ as a group of initial states. Low-lying states with different $n$ are well separated in energy (much more than the halfwidths of the lines) hence the transitions described by $\left\langle n k\left|T_{a}^{\prime}(t)\right| n k^{\prime}\right\rangle$ may usually be neglected. However, for large $n$ the levels are closer together and some of the higher series members may overlap; in this case it may be necessary to include states with different $n$ in the group of initial states.

In section (2.2) the no-quenching assumption was made by setting $\left\langle n h\left|T_{a}^{\prime}(t)\right| n^{\prime} h^{\prime}\right\rangle$ to zero. This neglects the quenching terms which would be added to eq (18) as discussed above, however, it also neglects the influence which $\left\langle n h\left|T_{a}^{\prime}(t)\right| n^{\prime} \hbar^{\prime}\right\rangle$ has on $\left\langle n h\left|T_{a}^{\prime}\right| n h^{\prime}\right\rangle$. That is, even if a line is isolated in the sense that the quenching terms which would be added to eq (18) are negligible, it may still be necessary to consider the influence of the inelastic transitions $|n h\rangle \rightarrow\left|n^{\prime} h^{\prime}\right\rangle$ when calculating $\left\langle n k\left|T_{a}^{\prime}\right| n h^{\prime}\right\rangle$. To show when this influence is also negligible we consider the differential equation for $T_{a}^{\prime}(t)$, eq (6). This equation may be written in the form

$$
\begin{aligned}
& i \hbar \frac{\partial}{\partial t}\left\langle n h\left|T_{a}^{\prime}(t)\right| n h^{\prime}\right\rangle \\
& =\sum_{h^{\prime \prime}}\left\langle n h|e| \mathscr{E} Z+V_{e}(t) \mid n h^{\prime \prime}\right\rangle\left\langle n h^{\prime \prime}\left|T_{a}^{\prime}(t)\right| n h^{\prime}\right\rangle \\
& \quad+\sum_{n^{\prime \prime} \neq n} \sum_{h^{\prime \prime}} \exp \left\{i t \omega_{n n^{\prime \prime}}\right\}\langle n h| e \mathscr{E} Z \\
& \quad+V_{e}(t)\left|n^{\prime \prime} h^{\prime \prime}\right\rangle\left\langle n^{\prime \prime} h^{\prime \prime}\left|T_{a}^{\prime}(t)\right| n h^{\prime}\right\rangle,
\end{aligned}
$$

where $\omega_{\prime \prime \prime \prime}=\left(E_{\prime \prime}-E_{\prime^{\prime \prime}}\right) / \hbar$. From this equation we see that setting $\left\langle n k\left|T_{a}\right| n^{\prime} k^{\prime}\right\rangle$ to zero simply neglects the influence which the "off-diagonal" matrix elements of $\left[e \mathscr{E} Z+V_{e}(t)\right]$ may have on $\left\langle\right.$ nt $\left|T_{a}\right|$ nht' $\rangle$. If eq (78) is solved in the usual iterative manner [7] by integrating over $t$, the term $\exp \left(i t \omega_{\text {,... }}\right)$ will oscillate rapidly and contribute essentially nothing for $t>\left(1 / \omega_{\ldots, \ldots}\right)$. This indicates that the $\left\langle n k|Z| n^{\prime \prime} k^{\prime \prime}\right\rangle$ terms will be negligible compared with the $\left\langle\left.{ }_{n}|Z| Z\right|_{n}{ }^{\prime}\right\rangle$ terms unless $t<\left(1 / \omega_{\ldots, \ldots}\right)$. Since the time of interest, $1 / \Delta \omega$, is much larger than $\left(1 / \omega_{\prime \prime \prime \prime \prime)}\right)$ for isolated lines, we may neglect the terms involving "off-diagonal" $Z$ matrix elements. The same results apply for the terms involving "off-diagonal" elements of $V_{e}(t)$ unless $t>\tau$. If $t>\tau$, a similar argument indicates that the "off-diagonal" $V_{e}(t)$ terms are negligible only when 
$\tau \omega_{\text {,., }}>1$. In this case we use $\tau \simeq \rho_{0} / v_{\text {av }}$ (the velocity distribution is sharply peaked about $v_{\text {av }}$ ) and we see that the condition $\tau \omega_{\text {...," }}>1$ is violated only when $\rho_{0} \leq\left(\hbar v_{\text {av }} / \Delta E_{\prime^{\prime \prime \prime}}\right)$ where $\Delta E_{\prime^{\prime \prime \prime}}=\left(E_{\prime \prime}-E_{\prime^{\prime \prime}}\right)$. Substituting this value of $\rho_{0}$ into $(V \tau / \hbar) \simeq\left(e^{2} R_{0} / \hbar \rho_{0} v_{\mathrm{av}}\right)$ (recall that $V \simeq e^{2} R_{0} / \rho_{0}^{2}$ for a hydrogen plasma) we find $(V \tau / \hbar) \simeq\left(e^{2} R_{0} \Delta E_{\ldots,{ }^{\prime \prime}} / \hbar^{2} v_{\text {av }}^{2}\right)$. Using $v_{\text {av }}^{2} \simeq(3 k T / m)$ and $R_{0} \simeq\left(3 n^{2} a_{0} / 2\right)$, where $a_{0}=\hbar^{2} / m e^{2}$, this becomes $(V \tau / \hbar) \geq\left(n^{2} \Delta E_{\ldots, "} / 2 k T\right)$. If we use $\Delta E_{\ldots, "}=E_{x} \mid(1 / n)^{2}$ $-\left(1 / r^{\prime \prime}\right)^{2} \mid$, where $E_{x}=13.6 \mathrm{ev}$. is the ionization energy of hydrogen, we find that the off diagonal-terms are important only when

$$
(V \tau / \hbar) \gtrsim\left(E_{x} / 2 k T\right)\left|1-\left(n / n^{\prime \prime}\right)^{2}\right| .
$$

Since $E_{x} \gg k T$ one usually has $\left(E_{x} / 2 k T\right)\left|1-\left(n / n^{\prime \prime}\right)^{2}\right|>1$ and, in this case, eq (79) indicates that the $\left\langle n^{\prime \prime} k^{\prime \prime}\left|T_{a}^{\prime}\right| n h^{\prime}\right\rangle$ terms in eq (78) are important only during strong collisions (in which case $T_{a}^{\prime}$ averages to zero, as discussed in sec. 7 of $\mathrm{I}$, so no error results). For some higher series members (very large $n$ and $n^{\prime \prime}$ ) it can happen that $\left(E_{x} / 2 k T\right)\left|1-\left(n / n^{\prime \prime}\right)^{2}\right|<1$, and eq (79) can be satisfied even for weak collisions. In this case, the "off-diagonal" terms in eq (78) must be retained when calculating $\left\langle n h\left|T_{a}^{\prime}\right| n h^{\prime}\right\rangle$. To see when this occurs, we consider the case where $n^{\prime \prime}=n \pm 1$ and $n \gg 1$. In this case the quantity $\left(E_{\infty} / 2 k T\right)\left|1-\left(n / \iota^{\prime \prime}\right)^{2}\right|$ will be less than unity when

$$
n>\left(E_{x} / k T\right) \text {. }
$$

Thus, for lines involving $n>\left(E_{x} / k T\right)$, we may not be able to neglect the influence of inelastic transitions between states having different principal quantum numbers when calculating $\left\langle n h\left|T_{a}^{\prime}\right| n k^{\prime}\right\rangle$. This effect is usually negligible for hydrogen lines from laboratory plasmas because, for these plasmas, the lines generally begin to merge (overlap) when eq (80) is satisfied. For some astrophysical applications [27] (and for lines other than hydrogen lines) this effect may be very important.

When the differential equation for the matrix elements of $T_{a l}^{\prime}(t)$ in the no-quenching approximation, eq (10), was written as an operator equation, eq (11), it was necessary to introduce a projection operator $P$ that picks out the part of an operator which is "diagonal" in $n$. Although $P$ operates on $Z$ and $V_{e}(t)$, this does not imply that the "off-diagonal" elements of these operators are small (indeed they are about the same order of magnitude as the "diagonal" elements). The appearance of $P$ in eq (11) merely indicates that the "off-diagonal" parts of $Z$ and $V_{e}(t)$ have a negligible influence on $T_{a \prime}^{\prime}$ in the noquenching approximation. The influence of this projection operator is implicit in most line broadening theories since matrix elements of $Z$ and $V$ are usually taken only between states having the same principal quantum numbers; we have introduced $P$ explicitly in order to avoid errors in handling $T_{a}^{\prime}(t)$ and to clarify subsequent approximations. For example, the only operator in $V_{e}(t)$ is the atomic position operator $\mathbf{R}$ and one may therefore commute
$Z$ with $V_{e}(t)$; this commutation is not possible with $P Z$ and $P V_{e}(t)$, in fact, the matrix elements of the commutator $\left[P Z, P V_{e}(t)\right]$ are about the same order of magnitude as the matrix elements of $P Z$ and $P V_{e}(t)$. If the influence of $P$ is only implicit, it is possible to make serious errors by commuting $Z$ with $V_{e}(t)$ when these operators appear in $T_{a l}^{\prime}(t)$.

Since $P Z$ does not commute with $P V_{e}(t)$, the formal solution, $T_{a}^{\prime}(t)$, of eq (11) is a complicated timeordered exponential. Although this operator has a rather simple form for the quasi-static region of the line wings (see sec. 3.4), it was necessary to introduce simplifying approximations in order to evaluate the rest of a line profile. The first of these was the impact approximation which assumes that most collisions can be described by a second order perturbation expansion of $U_{a}(t)$. Strong collisions $(V \tau / \hbar)>1$, for which this expansion breaks down, are treated by some other method such as an impact parameter cutoff. With this approximation, it is possible to write $U_{a}(t)$ in the product form $\mathscr{O}_{0} \Pi_{j} U_{j}(t)$ where each $U_{j}(t)$ is the time development operator for a single electron-atom collision. It is difficult to give a useful validity criterion for the impact approximation; the validity criterion which is usually given [3], $\tau \Delta \omega<1$, is based on the completed collision assumption which often accompanies the impact approximation when it is used in the impact theory (see secs. 6 and 7.3). Since the impact approximation is also used in the one-electron theory, we have drawn a distinction between the impact approximation and the impact theory. The impact approximation, as we have stated it, will be valid when strong collisions are well separated in time. If we define a strong collision frequency, $\nu_{s}$, and a maximum duration for a strong collision, $\tau_{s}$, this condition may be given by

$$
\tau_{s} \nu_{s} \ll 1 .
$$

If all collisions were strong, $\nu_{s}$ would be on the order of the halfwidth, $\Delta \omega_{1 / 2}$, of the spectral line. For hydrogen plasmas we have $\nu_{s}<\Delta \omega_{1 / 2}$. The duration of a strong collision may be estimated by using $(V \tau / \hbar) \simeq\left(e^{2} R_{0} /\right.$ $\left.\hbar \rho_{0} v_{\mathrm{av}}\right) \geq 1$ or $\rho_{0} \lesssim\left(e^{2} R_{0} / \hbar v_{\mathrm{av}}\right)$ which gives $\tau_{s} \simeq\left(\rho_{0} / v_{\mathrm{av}}\right)$ $\leqslant\left(e^{2} R_{0} / \hbar v_{\text {av }}^{2}\right)=\left(\widetilde{1} / \Delta \omega_{c}\right)$, where $\Delta \omega_{c}$ is the Weisskopf frequency for electrons. Using $v_{\mathrm{av}}^{2} \simeq(3 k T / m)$ and $R_{0}=\left(3 n^{2} a_{0} / 2\right)$ where $a_{0}=\left(\hbar^{2} / m e^{2}\right)$, we have $\tau_{s}$ $\leq\left(n^{2} \hbar / k T\right)$ and $\tau_{s} \nu_{s}<n^{2}\left(\hbar \Delta \omega_{1 / 2} / k T\right)$. Since $n^{2}\left(\hbar \Delta \omega_{1 / 2} /\right.$ $\widetilde{k} T) \ll 1$ for most hydrogen plasmas, we expect the impact approximation to be valid for the electrons.

\subsection{Discussion of the Impact Theory}

In the usual derivation of the impact theory $[2,3]$ one calculates the change in $\left\langle U_{a}(t \mid 0)\right\rangle$ during some time $\Delta t$ (recall that $\left.U_{a}(t) \equiv U_{a}(t \mid 0)\right)$. This change is given by

$$
\begin{aligned}
\Delta\left\langle U_{a}(t \mid 0)\right\rangle & =\left\langle U_{a}(t+\Delta t \mid 0)-U_{a}(t \mid 0)\right\rangle \\
& =\left\langle\left[U_{a}(t+\Delta t \mid t)-1\right] U_{a}(t \mid 0)\right\rangle .
\end{aligned}
$$

It is assumed that: (1) $\Delta t$ is large enough that the two factors on the right are statistically independent and 
may thus be averaged separately, and (2) $\Delta t$ is small enough that the difference equation, eq (82), may be replaced by the differential equation (see eq (4.30) of ref. [2], eq (38) of ref. [3] or eq (2.8) of ref. [20])

$$
\frac{d}{d t}\left\langle U_{a}(t)\right\rangle=\left[\exp \left(i t H_{a} / \hbar\right) \Phi_{a} \exp \left(-i t H_{a} / \hbar\right)\right]\left\langle U_{a}(t)\right\rangle
$$

which is then solved for $\left\langle U_{a}(t)\right\rangle$ ( $\Phi_{a}$ is given in eq (71)). In this derivation, assumption (1) requires $\Delta t>\tau$ and assumption (2) requires $\Delta t \ll t$ (as well as the average collision being weak; cf. pp. 508 and 529 of ref. [3]); together these assumptions require $t \gg \tau$ which is a mathematical statement of the completed collision assumption (cf. eq (68)). Thus we see that the completed collision assumption is an integral part of the usual derivation of the impact theory and the removal of this approximation would require a complete revision of the derivation. This is the reason one frequently sees the condition $t \gg \tau$ or $\tau \Delta \omega \ll 1$ given as the validity criterion for the impact approximation [3].

In our derivation of the impact theory we first used the impact approximation to write $U_{a}(t)$ in the product form $\mathscr{O}_{0} \Pi_{j} U_{j}(t)$ and we obtained a consistent correlation function expansion, eq $(50)$, for $\left\langle U_{a}(t)\right\rangle \equiv F(t)$. To obtain the usual results of the impact theory, we assumed that the electron-electron correlations could be approximated by impact parameter cutoffs, we used the completed collision assumption and we assumed that $\tau \Phi_{a}<1$. It was also noted that numerical calculations are frequently simplified by approximating the influence of the ion field splitting on the electron broadening (i.e., the factors $\exp (i s \mathscr{Z})$ in eq (67)) with an impact parameter cutoff $\rho_{\max }=v_{0} \mid \omega_{\text {mkt }}$, nh' which depends on the ion field strength. While this approximation does not affect the region of validity of the theory, it would nonetheless be desirable to improve on this approximation to insure more accurate calculations. It is also interesting to note that the condition $\tau \Phi_{a}<1$ is simply a manifestation of the requirement that the impact approximation is satisfied (this was discussed in sec. 7.2). To show this, we use eq (71) and estimate the magnitude of $\Phi_{a}$ by

$$
\Phi_{a}=n[S-1]_{\mathrm{av}} \simeq n(V \tau / \hbar)_{\mathrm{av}}
$$

where the subscript av refers to an integral over the collision variables $\rho_{0}, v_{0}$, and $\Omega$ (cf. eqs (64) and (A.5)). Since $2 \pi n \rho_{0} d \rho_{0} v_{0}^{3} W\left(v_{0}\right) d v_{0} d \Omega=d \nu$ where $\nu$ denotes the frequency of collisions described by $\rho_{0}, v_{0}$, and $\Omega$, we see that $n(V \tau / \hbar)_{\mathrm{av}}=\int(V \tau / \hbar) d \nu=(\bar{V} / \hbar)$ where $\bar{V}$ denotes the average value of $V$. The statement $\tau \Phi_{a}<1$ now becomes $(\vec{V} \tau / \hbar)<1$, which is satisfied if the average collision is weak and if strong collisions $(V \tau / \hbar>1)$ are well separated in time. This shows that the condition $\tau \Phi_{a}<1$ is always satisfied when the impact approximation is valid.

One potentially useful feature of our approach is that it permits a consistent improvement of the impact theory within the framework of the classical path methods. That is, the impact parameter cutoffs, the completed collision assumption and the second order expansion of $U_{j}(t)$ were needed only to simplify the calculation of eq (50) and it would be possible to correct these approximations without having to rewrite the theory. In fact, the impact parameter cutoffs have already been corrected to some extent by means of a more consistent treatment of correlation functions $[22,23]$.

The correction of the completed collision assumption is a matter of some importance because, if we estimate the collision duration time by $\left(\lambda_{D} / v_{\mathrm{av}}\right)=\left(1 / \omega_{p}\right)$, the region of validity for this approximation, eq (66), is given by

$$
\Delta \omega<\omega_{p}
$$

That is, an impact theory with a completed collision assumption can be used only for values of $\Delta \omega$ smaller than the electron plasma frequency. It has been shown that the completed collision assumption may be corrected, in the line wings, by means of the Lewis cutoff [21]. This cutoff may be obtained from eqs (61) and (62) by expanding $F(t)$ to second order in $V(t)$ and performing the Fourier transform as stated in eq (18) (cf. eqs (6) and (17) of ref. [21]).

Since the derivation of the Lewis cutoff is based on an expansion of $F(t)$ which is valid only in the line wings, the use of this cutoff in the impact theory raises some doubts as to the validity of such a "modified" impact theory in the line center. These doubts may be dispelled to some extent by means of a comparison with the results of the quantum mechanical relaxation theory which does not make a completed collision assumption. In reference [22] it is shown that the results of the relaxation theory essentially reproduce the behavior of a Lewis cutoff, and these results are not restricted to the line wings. In spite of this useful comparison, it would nonetheless be desirable to confirm this behavior within the framework of a classical path theory. In this regard, it is important to note that the completed collision assumption is not an inherent part of our derivation of the impact theory. This approximation was introduced simply to compare with the results of the usual derivation. It would thus be possible, in principle, to calculate $F(t)$ from eqs $(61)$ and (62) without making a completed collision assumption. This has in fact been done, to second order, $[28]$, and it is found that the results are identical with the results of the relaxation theory. It thus appears that a "modified" impact theory which does not make a completed collision assumption (such as ref. [28]), or a theory which corrects this assumption by means of a Lewis cutoff, will have a wider range of applicability than that stated in eq (84).

If the completed collision assumption is not made (or if it can be corrected by some approximation such as the Lewis cutoff), the only serious limitation on the applicability of such a "modified" impact theory comes from the second order expansion of $U_{j}(t)$. This expansion should be valid whenever the quantity $(i / \hbar) \int V\left(t^{\prime}\right) d t^{\prime}$ is small compared with unity. For completed collisions this quantity was estimated (cf. eq $(\mathrm{I}-68))$ by $(V \tau / \hbar)$ and the expansion was justified 
except for strong collisions. For collisions which cannot be completed during the time of interest (i.e., collisions for which $\tau \Delta \omega>1)$, we have $(1 / \hbar)$ $\int V\left(t^{\prime}\right) d t \simeq(V / \hbar \Delta \omega)$ and it remains to be seen whether this is small or not. This situation may arise in the far wings of a line where the electron-atom collisions take on a quasi-static aspect (cf. sec. 3.4). In this region, the intensity at a point $\Delta \omega$ is primarily determined by those interactions which produce a Stark splitting $\hbar \Delta \omega$; that is, by those interactions for which $V \simeq \hbar \Delta \omega$ (see p. 23 of ref. [4]). For these quasi-static interactions we have $(1 / \hbar) \int V\left(t^{\prime}\right) d t^{\prime} \simeq(V / \hbar \Delta \omega) \simeq 1$ and the series expansion of $U_{j}(t)$ breaks down [29]. To determine where this breakdown occurs, we use $\tau \simeq\left(\rho_{0} / v_{\mathrm{av}}\right)\left(v_{0} \simeq v_{\mathrm{av}}\right.$ for neutral radiators $)$ and $\tau \Delta \omega \gtrsim 1$ to obtain $\rho_{0} \gtrsim\left(v_{\text {av }} / \Delta \omega\right)$; substituting this result in $\hbar \Delta \omega \simeq V \simeq e^{2} R_{0} / \rho_{0}^{2}$, we obtain $\Delta \omega \simeq\left(\hbar v_{\mathrm{av}}^{2} / e^{2} R_{0}\right)=\Delta \omega_{c}$ where $\Delta \omega_{c}$ is the Weisskopf frequency for electrons. Using $v_{\text {av }}=(3 k T / m)^{1 / 2}$ and $a_{0}=\left(\hbar^{2} / m e^{2}\right)$, we have $\Delta \omega_{c}=(3 k T / \hbar)\left(a_{0} / R_{0}\right)$; thus we see that the second order expansion of $U_{j}(t)$ limits the application of the impact theory to the region

$$
0 \lesssim \Delta \omega \ll \Delta \omega_{c}=(3 k T / \hbar)\left(a_{0} / R_{0}\right) .
$$

In principle this range of applicability could be extended by using the full exponential for $U_{j}(t)$ rather than its series expansion. In this regard it should be noted that the derivation of the Lewis cutoff is based on the second order expansion and, if this expansion is to be improved, it may be necessary to improve the Lewis cutoff as well.

\subsection{Discussion of the One-Electron Theory}

In the previous section it was shown that the impact theory is limited to the center region of a line profile because of the second order expansion of $U_{j}(t)$. The one-electron theory is designed to be valid in the line wings and it is expected that its region of validity will overlap with that of the impact theory so that the complete line profile may be described without ambiguity. To insure that the one-electron theory will be valid in the line wings, we used the exponential form of $U_{j}(t)$ which is valid in the wings. In the derivation of this exponential form it was assumed that both the ion field splitting and the time ordering operator could be neglected.

In section 3.3 it was shown that the time ordering operator is not necessary if the second order expansion of $U_{j}(t)$ is justified, and in section 3.4 it was shown that time ordering is not necessary for quasi-static interactions. It thus appears that the only errors resulting from the neglect of time ordering will come from the strong collisions. Since the average effect of strong collisions is to reduce $U_{j}(t)$ to zero (see sec. 7 of $I$ ), the errors made in these collisions should have a negligible effect on $\left\langle U_{j}(t)\right\rangle$.

To find out when we may neglect the exponential $\exp \left[\right.$ ite $\left.\mathscr{E}\left(Z_{\text {, th }}-Z_{\text {, } \hbar_{i}}\right) / \hbar\right]$ which appears in the operator $U_{n}(t)$ used by the one-electron theory, eq (28), we consider a cutoff that is similar to the cut off $v / \omega$, th, , th which approximates this exponential in the impact theory (see eq (75)). Using $t=1 / \Delta \omega$ and $\omega \ldots, \ldots(\mathscr{E})$ $=e \mathscr{E}\left(Z, \ldots-Z, h_{i}\right) i \hbar$, we note that this exponential is essentially unity when $\left(\omega, \ldots, k^{\prime} / \Delta \omega\right)<1$; when $\left(\omega, h_{1}, \ldots, \mid \Delta \omega\right) \gg 1$ the exponential oscillates rapidly and there is only a negligible contribution from $U_{a}(t)$. Since $U_{n}(t)$ appears under a microfield average, we will consider a cutoff $\mathscr{E}_{c}=\hbar \Delta \omega / e\left(Z_{, \ldots}-Z_{, \ldots}\right)$ in the range of this microfield average. We replace the exponential by unity and average over ion field strengths $\mathscr{E}<\mathscr{E}_{i} ;$ this cutoff replaces the integrand by zero when $(\omega, \ldots, \ldots, \mid \Delta \omega)>1$, thus approximating the effect of the rapidly oscillating exponentials, and when $(\omega, \ldots, \ldots, \mid \Delta \omega)<1$, the exponentials are effectively replaced by unity. This cutoff has no effect on the static ion broadening because, in the one-electron theory, the line shape is a sum of two terms (cf. eq (51)) and the term which describes the ion broadening (the $\mathscr{P}(\hbar \Delta \omega / Z, \ldots)$ term) is not affected by the microfield average of the electron broadening term (the $F_{1}(t)$ term) where the cutoff is applied. If it should happen that $\mathscr{E}_{.} \gg \mathscr{E}_{\text {av }}$, we may as well ignore the cutoff and average over $0 \leqslant \mathscr{E} \leqslant \infty$ because $\mathscr{P}(\mathscr{E})$ will be negligibly small for $\mathscr{E}>\mathscr{E}$. . That is, the exponential may be replaced by unity for all $\mathscr{E}$ whenever $\mathscr{E}_{r}=\hbar \Delta \omega / e\left(Z_{, h}-Z_{, h_{i}}\right)>\mathscr{E}_{\text {av }}$ or, as it was stated in eq (29), whenever

$$
\Delta \omega>e \mathscr{E}_{\text {av }}\left(Z_{\text {,h }}-Z_{\text {, hi }}\right) / \hbar
$$

It should be noted that, as a practical estimate of the region of validity for neglecting the effect of the ion field on electron broadening, eq (86), is usually too restrictive. For higher lines (large $n$ ) which have a large number of Stark components (allowed $|n h\rangle \rightarrow|1\rangle$ transitions) a more realistic estimate is obtained by considering the average Stark splitting

$$
\Delta \omega>e \mathscr{C}_{\mathrm{av}} Z_{\mathrm{av}} / \hbar
$$

which is usually on the order of the halfwidth [30]. The use of $Z_{\text {ar }}$ rather than the maximum value of $\left(Z, \ldots-Z, Z_{1}\right)$ is more appropriate for the higher lines because, for the larger values of $Z_{\text {, hh }}$, the quantity $e \mathscr{E}_{\text {av }} Z_{\text {, }} / \hbar$ corresponds to a very large frequency and the electron broadening is negligible for some values of $\Delta \omega$ less than $e \mathscr{E}_{\text {ar }} Z, \ldots / \hbar$ (for large $Z_{\text {,h, }} \mathscr{P}\left(\hbar \Delta \omega / e Z_{, h}\right.$ ) is much larger than the electron broadening term for a wide range of $\Delta \omega$ which are less than $e \mathscr{E}_{\text {ax }} Z$, $\left.t / \hbar\right)$. Since we wish to use a classical potential in $U_{j}(t)$ to describe the quasi-static interactions, it is necessary to justify the use of the classical wavepackets which give rise to a classical potential (in sec. 8.3 of $\mathrm{I}$, we justified the use of classical wavepackets only for dynamic perturbers). In section 8.3 of I, it was shown that we may represent the perturbers by nonoverlapping classical wavepackets of width $\Delta x$ and momentum uncertainly $\Delta p \ll p$ if

$$
\chi_{\text {av }} \ll \Delta x \ll n^{-1 / 3} .
$$

Since we have $X_{\mathrm{av}} \ll n^{-1 / 3}$ for most problems of interest, this criterion is easily met. We thus need only 
show that the radiator and perturber wave functions do not overlap during the time of interest. Using the notation of section 8.3 in I, this criterion may be written

$$
\left[\Delta x+(t \Delta p / m)+R_{0}\right]<\rho_{0}
$$

where $R_{0} \simeq n^{2} a_{0}=n^{2} \hbar^{2} / m e^{2}$ denotes the effective extent of the radiator wave function. Using $\Delta p \ll p$ and $t<\tau=\left(\rho_{0} / v_{0}\right)$, we see that $(t \Delta p / m) \ll \rho_{0}$ hence the $(t \Delta p / m)$ term in eq (89) may be dropped. Using $V \simeq \hbar \Delta \omega$ (for quasi-static interactions) and $V \simeq e^{2} R_{0} / \rho_{0}^{2}$ we obtain $\rho_{0}^{2} \simeq\left(e^{2} R_{0} / \hbar \Delta \omega\right)=R_{0}^{2}\left(e^{2} / r^{2} a_{0}\right) \quad(1 / \hbar \Delta \omega)$ $=R_{0}^{2}\left(2 E_{x} / r^{2} \hbar \Delta \omega\right)$ where $E_{\infty}=e^{2} / 2 a_{0}$ is the ionization energy. Since $\hbar \Delta \omega \ll E_{x} / n^{2}$, we see that $R_{0} \ll \rho_{0}$ hence the $R_{0}$ term in eq (89) may also be dropped and we need only show that

$$
\Delta x<\rho_{0} .
$$

Again using the value $\rho_{0}^{2} \simeq\left(e^{2} R_{1} \hbar \Delta \omega\right)$ given above, as well as $\chi_{\mathrm{a} \overline{\mathrm{v}}}^{2}=\left(\hbar^{2} / 3 m k^{2} T\right)$ and $a_{0}=\hbar^{2} / m e^{2}$, we find that $\rho_{0}^{2}=\chi_{\mathrm{av}}^{2}(k T / \hbar \Delta \omega)\left(R_{0} / a_{0}\right)$. Since $R_{0}>a_{0}$ and $\hbar \Delta \omega \ll k T$, we see that $\rho_{0} \gg \chi_{\text {av }}$ hence it is possible to find a $\Delta x$ which will satisfy both eq (90) and eq (88). The use of a classical potential function is thus justified for quasi-static electron-atom interactions.

The results of the one-electron theory are based on the approximation $F(t) \simeq 1+F_{1}(t)$ which treats the electron-atom interactions as though they are independent of one another. In section 4 it was arqued that this is probably a good approximation, if the times of interest are shorter than the mean free time between strong collisions. To obtain a validity criterion for this approximation one sometimes identifies the collision frequency with the halfwidth $\Delta \omega_{1 / 2}$ for the line under consideration; the requirement that the time of interest, $1 / \Delta \omega$, be less than the mean free time between collisions is then written [3]

$$
\Delta \omega>\Delta \omega_{1 / 2}
$$

Although this argument is somewhat loose, it will be shown that eq (91) actually does provide a useful statement of the region of validity for the one-electron theory.

To derive a validity criterion for the one-electron theroy, we note that the approximate $F(t)$ used in this theory, eq (51), is formally the same as an expansion to lowest order in $h_{1}(t)$ of the approximate $F(t)$ used in the impact theory, eq (61). That is, the single particle approximation is simply a small $h_{1}(t)$ approximation which is valid when $n\left|h_{1}(t)\right|<1$. From the definition of $h_{1}(t)$ (see eq (62)) it is clear that $n\left|h_{1}(t)\right|<1$ for small $t$. For large $t$ the impact theory is valid and we use eqs (66) and (71) to obtain $n\left|h_{1}(t)\right| \leqslant t \Phi_{\text {t }}$. Replacing $t$ by the time of interest, $1 / \Delta \omega$, the validity criterion $n\left|h_{1}(t)\right|<1$ becomes

$$
\Delta \omega>\Phi_{a} .
$$

Thus the one-electron theory should be valid when both eqs (86) and (92) are satisfied (or, for higher lines, when eqs (87) and (92) are satisfied).
If the average ion field splitting is small. ( $\left(e \mathscr{E}_{\text {av }} Z, t_{i} / \hbar\right)$ $<\Phi_{a}$, or if the linear Stark effect due to the ion field does influence the line shape (e.g., isolated lines), the halfwidth is determined [30] by the matrix elements of $\Phi_{a}$ (see sec. 4.7 of ref. [2]). In this case, the region of validity is given by eq (91) as $\Delta \omega>\Delta \omega_{1 / 2}$.

For lines where the average ion field splitting is large, $\left(e \mathscr{E}_{\mathrm{av}} Z_{\mathrm{av}} / \hbar\right)>\Phi_{\text {" }}$ (e.g., higher hydrogen lines) the halfwidth is determined primarily by ion broadening [30| and $\Delta \omega_{1 / 2} \simeq\left(e \mathscr{C}_{\mathrm{av}} Z_{\mathrm{av}} / \hbar\right)$. In this case eq (87) is more restrictive than eq (92) and the region of validity is again given by $\Delta \omega>\Delta \omega_{1 / 2}$.

The low lying hydrogen lines are determined by a small number of Stark components and, for many of these lines, a discussion of the halfwidth is of little value; lines such as $L y-\beta$ and $H-\beta$ do not even have a well-defined halfwidth. Lines such as Ly- $\alpha$ and $H-\alpha$ have a very intense Stark component which is not shifted by linear Stark effect and the halfwidths of these lines are determined primarily by the electron broadening of this unshifted component. In such a case, eq (92) gives $\Delta \omega>\Delta \omega_{1 / 2}$. However, the remaining Stark components are very important in determining the line profile outside of the relatively narrow central component and, for these shifted components, eq (86) becomes more restrictive than the condition $\Delta \omega>\Delta \omega_{1 / 2}$ obtained from eq (92). It should be noted that eq (86) is probably not very much more restrictive than the condition $\Delta \omega>\Delta \omega_{1 / 2}$; for example, in the cases where Ly- $\alpha$ has been studied experimentally $[31,32]$, eq (86) gives approximately $\Delta \omega>\omega_{p} / 10$ and $\omega_{p} / 10$ differs very little from $\Delta \omega_{1 / 2}$.

From the above discussion we conclude that the one-electron theory will be valid in the region $\Delta \omega>\Delta \omega_{1 / 2}$ for isolated lines and for overlapping lines which have a large number of Stark components. For overlapping lines with only a few Stark components, the condition $\Delta \omega>\Delta \omega_{1 / 2}$ may no longer be applicable and one should consider eqs (86) and (92) for each Stark component.

\section{Comparison of the Impact and One-Electron Theories}

The calculations in both the impact and one-electron theories are based on binary collisions. The oneelectron theory treats the electron-atom interactions as though their effects are additive and for this reason the one-electron theory is limited to the line wings (short times of interest) whereas the impact theory is not. In principle, the impact theory (with a Lewis cutoff) should be valid over most of the line profile; however, in practice it is usually necessary to expand the time development operator, $U_{j}(t)$, in order to make practical calculations, and it is this expansion which limits the impact theory to the center region of a line profile.

It was shown that the series expansion of $U_{j}(t)$ could not adequately describe either the strong collisions, $(V \tau / h) \gtrsim 1$, or the quasi-static interactions, $(i / h) \int V(t) d t \simeq 1$, which are important in the line wings. In our version of the one-electron theory this 
difficulty is avoided through the use of an exponential expression for $U_{j}(t)$ (previous versions of the oneelectron theory have used either a completed collision assumption [5] or a series expansion [3] of $U_{j}(t)$ hence they cannot be valid in the quasi-static wings). While this exponential form is not rigorously correct for strong collisions it does have the following useful properties:

(1) It is correct for weak collisions, $(V \tau / \hbar)<1$, since it reproduces the series expansion for such interactions.

(2) It is correct for quasi-static interactions whereas the series expansion is not.

(3) For strong collisions, $(V \tau / \hbar) \geq 1$, the exponential would give a much more realistic approximation to the exact $U_{j}(t)$ than the series expansion because the exponential retains unitarity (i.e., no transition probability can exceed unity). Thus, if one chose to use classical path methods for calculating $U_{j}(t)$, the strong collision cutoff discussed in section 7 of I, would be essentially reproduced (i.e., the exponential would oscillate rapidly and contribute essentially nothing for strong collisions). The errors resulting from this treatment of strong collisions should be negligible because the averaged $U_{j}(t)$ for strong collisions is essentially zero.

It is interesting to note that if we use a second order expansion of $U_{j}(t)$ in the one-electron theory, we obtain the asymptotic wing expansion which is used in deriving the Lewis cutoff [21]. That is, the Lewis cutoff, which is applied to the impact theory in an ad hoc manner, is an integral part of the one-electron theory.

Since the Weisskopf frequency, $\Delta \omega_{c}$, for electrons is generally much greater than the halfwidth, there should be a broad range of overlap between the regions of validity $0 \leqslant \Delta \omega<\Delta \omega_{c}$ and $\Delta \omega_{1 / 2}<\Delta \omega$ for the impact and one-electron theories. Nevertheless, in our derivation of the impact and one-electron theories we have tried to stress the formal connection between the two in order to provide a better understanding of their similarities as well as their differences. We feel that this is particularly important since it would be desirable to formulate a classical path theory which is valid in the line center as the impact theory is, and which makes a smooth transition to the line wings as the one-electron theory does. It is hoped that the present approach will make it possible to remove some of the approximations which currently limit these classical path theories.

\section{Appendix. Collision Variables}

We wish to use the natural collision coordinates in evaluating the integral

$$
\begin{aligned}
& F_{1}(t)=n \int d \mathbf{v}_{1} W\left(v_{1}\right) \int d \mathbf{x}_{1} \\
& \quad\left[\exp \left\{-(i / \hbar) \int_{0}^{t} P V_{1}\left(\mathbf{R}, \mathbf{x}_{1}+\mathbf{v}_{1} t^{\prime}\right) d t^{\prime}\right\}-1\right]
\end{aligned}
$$

where
$V_{1}\left(\mathbf{R}, \mathbf{x}_{1}+\mathbf{v}_{1} t^{\prime}\right)=e^{2} \mathbf{R} \cdot\left(\mathbf{x}_{1}+\mathbf{v}_{1} t\right) /\left|\mathbf{x}_{1}+\mathbf{v}_{1} t\right|^{3}$.

For fixed values of $\mathbf{x}_{1}$ and $\mathbf{v}_{1}$, we note that the head of the vector $\left(\mathbf{x}_{1}+\mathbf{v}_{1} t^{\prime}\right)$ traces out a straight line if $t^{\prime}$ is varied from $-\infty$ to $+\infty$. We shall call this line $L\left(\mathbf{x}_{1}, \mathbf{v}_{1}\right)$. The integral over $t^{\prime}$, in eq (A.1), will cover a segment of this line. For fixed $\mathbf{x}_{1}$ and $\mathbf{v}_{1}$, we can define a vector $\boldsymbol{\rho}_{0}$ from the origin of coordinates to the closest point on $L$; this vector will be perpendicular to $\mathbf{v}_{1}$. We can also define a scalar $t_{0}$ by

$$
\mathbf{x}_{1}=\boldsymbol{\rho}_{0}+\mathbf{v}_{1} t_{0} .
$$

For a fixed $\mathbf{v}_{1}$, we see that, as $\mathbf{x}_{1}$ varies over all space, $\left|\mathbf{v}_{1}\right| t_{0}$ varies on the range $(-\infty,+\infty)$ and $\rho_{0}=\left|\boldsymbol{\rho}_{0}\right|$ varies on the range $(0, \infty)$. We may thus change variables from $\mathbf{x}_{1}$ to the cylindrical coordinates $\left(\rho_{0}, t_{0}, \alpha\right)$ where $\mathbf{v}_{1}$ defines the polar direction and $\alpha$ is an angle which ranges from 0 to $2 \pi$. The Jacobian for this transformation is such that $\mathbf{d} \mathbf{x}_{1} \rightarrow\left|v_{1}\right| \rho_{0} d \rho_{0} d t_{0} d \alpha$. In these coordinates the potential function becomes

$$
\begin{aligned}
V_{1}\left(\mathbf{R}, \boldsymbol{\rho}_{0}, \mathbf{v}_{1}, t^{\prime}+t_{0}\right)=e^{2} \mathbf{R} \\
\cdot\left[\boldsymbol{\rho}_{0}+\mathbf{v}_{1}\left(t^{\prime}+t_{0}\right)\right] /\left[\rho_{0}^{2}+v_{1}^{2}\left(t^{\prime}+t_{0}\right)^{2}\right]^{3 / 2} .
\end{aligned}
$$

We may express $d \mathbf{v}_{1}$ in spherical coordinates, $v_{0}^{2} d v_{0}$ $\sin \beta d \beta d \gamma$, where the angles $\beta$ and $\gamma$ denote the orientation of $\mathbf{v}_{1}$ relative to the direction of $\mathbf{R}$. Since $\boldsymbol{\rho}_{0}$ is defined to be orthogonal to $\mathbf{v}_{1}$, the integrals over the Euler angles $\alpha, \beta$, and $\gamma$ average over all possible orientations of the $\left(\boldsymbol{\rho}_{0}, \mathbf{v}_{1}\right)$ frame of reference (collision axes) relative to the direction of R. Equation (A.1) may now be written

$$
\begin{aligned}
& F_{1}(t)=n \int d \Omega \int_{0}^{\infty} v_{0}^{3} W\left(v_{0}\right) d v_{0} \int_{0}^{\infty} \rho_{0} d \rho_{0} \int_{-\infty}^{\infty} d t_{0} \\
& {\left[\exp \left\{-(i / \hbar) \int_{0}^{t} P V_{1}\left(\mathbf{R}, \boldsymbol{\rho}_{0}, \mathbf{v}_{0}, t^{\prime}+t_{0}\right) d t^{\prime}\right\}-1\right]}
\end{aligned}
$$

where $d \Omega=\sin \beta d \alpha d \beta d \gamma$.

It is clear that the line $L$ represents the trajectory of a perturbing electron, $v_{0}$ is the speed of the electron, $\rho_{0}$ is the impact parameter, and $t_{0}$ is some starting time for the collision (or, if the $t^{\prime}$ limits of integration are $(-\infty,+\infty)$ in a completed collision, $t_{0}$ may be regarded as the time of closest approach). The orthogonal "collision axes" to which this collision is referenced are $\boldsymbol{\rho}_{0}$ and $\mathbf{v}_{1}$. The Euler angles $\Omega$ denote the orientation of the collision plane relative to the direction of $\mathbf{R}$; the integral over $\Omega$ may therefore be interpreted as an average over all possible orientations of the electron trajectory.

\section{References and Notes}

[1] Smith, E. W., Vidal, C. R., and Cooper, J., J. Res. Nat. Bur. Stand. (U.S.), 73A (4) 389-404 (1969).

[2] Griem, H. R., Plasma Spectroscopy, chap. 4 (McGraw-Hill Book Co., Inc., New York, 1964).

[3] Baranger, M., Atomic and Molecular Processes, chap. 13, edited by D. Bates (Academic Press, Inc., New York, 1962). 
[4] Baranger, M., Rand Corporation Report No. (RM-2118-AEC) ASTIA Document No. (AD-150692) (Feb. 1958).

[5] Cooper, J., Phys. Rev. Letters 1 7, 991 (1966).

[6] Smith, E. W., and Hooper, C. F., Jr., Phys. Rev. 157, 126 (1967).

[7'] Schweber, Silvan S., An Introduction to Relativistic Quantum Field Theory, pp. 330-334 (Harper and Row Publishers, New York, 1962).

[8] Callaway, J., and Bauer, E., Phys. Rev. 140, Al072 (1965).

[9] Kolb, A. C., and Griem, H. R., Phys. Rev. 111, 514 (1962).

[10] If $H_{a}$ is to remain diagonal in $|n h\rangle$, it is possible to diagonalize $Z$ only "within a principal quantum number"; that is, one may simultaneously diagonalize both $H_{a}$ and $P Z$ but not $H_{a}$ and $Z$.

[11] Bethe, H., and Salpeter, E., Quantum Mechanics of One- and Two-Electron Atoms (Academic Press Inc., New York, 1957).

[12] Baranger, M., Phys. Rev. 111, 494 (1958).

[13] Griem, H. R., Kolb, A. C., and Shen, K. Y., Phys. Rev. 116 , 4 (1959).

[14] Magnus, W., Comm. Pure and Appl. Math. 7, 649 (1954) and Robinson, D. W., Helv. Phys. Acta. 36, 140 (1963).

[15] Baranger, M., and Mozer, B., Phys. Rev. 115, 521 (1959).

[16] Hill, T. L., Statistical Mechanics, chap. 6. (McGraw-Hill Book Co., Inc., New York, 1963).

[17] Ursell, H. D., Proc. Cambridge Phil. Soc. 23, 685 (1927); see also eq. (9) of ref. [15]).

[18] Edmonds, A. R., Angular Momentum in Quantum Mechanics (Princeton University Press, Princeton, New Jersey, 1960).

[19] Hughes, J. W. B., Proc. Phil. Soc. 91, 810 (1967).
[20] Griem, H. R., Baranger, M., Kolb, A. C., and Oertel, G. K., Phys. Rev. 125, 177 (1962).

[21] Lewis, M., Phys. Rev. 121, 501 (1960).

[22] Smith, E. W., Phys. Rev. 166, 102 (1968).

[23] Chappell, W. R., Cooper, J., and Smith, E. W., J. Quant. Spectry. Radiative Transfer 9, 149 (1969).

[24] Kepple, P., and Griem, H. R., Phys. Rev. 173, 317 (1968).

[25] Bezzerides, B., J. Quant. Spectry. Kadiative Transfer 7, 353 (1967).

[26] Griem, H. R., Phys. Rev. 140, A1140(1965).

[27] Griem, H. R., Ap. J. 148, 547 (1967).

[28] Smith, E. W., unpublished notes.

[29] It should be noted that some authors use the term strong collision to describe any interaction for which $(1 / \hbar) \int V(t) d t \gtrsim 1$ and, for this reason, quasi-static interactions are sometimes referred to as strong collisions.

[30] From eq (4-35) of ref. [2] we see that the line shape in the impact theory (which is valid in the line center) is roughly given by the real part of $\left[\Delta \omega-e Z \mathscr{E}_{\text {av }} / \hbar-i \Phi_{a}\right]^{-1}$. Thus, if the matrix elements of $\Phi_{a}$ are larger than $\left(e Z_{\text {av }} \mathscr{E}_{\text {av }} / \hbar\right)$ the halfwidth is on the order of $\Phi_{a}$. If $\Phi_{a}<\left(e Z_{\mathrm{av}} \mathscr{E}_{\mathrm{av}} / \hbar\right)$ as is usually the case, the halfwidth is determined by the average ion field splitting $\left(e Z_{\text {av }} \mathscr{E}_{\text {av }} / \hbar\right)$.

[31] Elton, R. C., and Griem, H. R., Phys. Rev. 135, A1550 (1964).

[32] Boldt, G., and Cooper, W. S., Z. Naturforsch. 19a, 968 (1964).

(Paper 73A4-559) 